\title{
Quasilinear Hyperbolic Systems
}

\section{Tai-Ping Liu*}

Institute of Physical Science and Technology, and Department of Mathematics, University of Maryland, College Park, Maryland 20742, USA

\begin{abstract}
We construct global solutions for quasilinear hyperbolic systems and study their asymptotic behaviors. The systems include models of gas flows in a variable area duct and flows with a moving source. Our analysis is based on a numerical scheme which generalizes the Glimm scheme for hyperbolic conservation laws.
\end{abstract}

We consider the initial value problem for quasilinear partial differential equations of the following form

$$
\begin{aligned}
\frac{\partial u}{\partial t}+\frac{\partial f(u)}{\partial x} & =g(x, u), \quad-\infty<x<\infty, \quad t \geqq 0, \\
u(x, 0) & =u_{0}(x), \quad-\infty<x<\infty .
\end{aligned}
$$

Here $u=u(x, t)$ is an $n$-vector, $f$ is a smooth $n$-vector-valued function of $u$, and $g$ and $\frac{\partial g}{\partial u}$ are piecewise continuous $n$-vector-valued function of $x$, and are continuous in $u$. System $(0.1)$ is assumed to be strictly hyperbolic, that is $\partial f(u) / \partial u$ has real and distinct eigenvalues $\lambda_{1}(u)<\lambda_{2}(u)<\ldots<\lambda_{n}(u)$ for each $u$. In general (0.1) and $(0.2)$ do not possess smooth solutions, and we look for weak solutions, that is, solutions satisfying

$$
\iint_{t \geqq 0}\left(u \frac{\partial \varphi}{\partial t}+f(u) \frac{\partial \varphi}{\partial x}-g(x, u) \varphi\right) d x d t+\int_{-\infty}^{\infty} u_{0}(x) \varphi(x, 0) d x=0
$$

for any smooth function $\varphi(x, t)$ with compact support in $t \geqq 0$. The purpose of this paper is to construct solutions for (0.1) and (0.2) and study their asymptotic behavior as the time variable $t$ tends to infinity.

* Partially supported by National Science Foundation Grant NSF MCS 78-2202 
When $g \equiv 0$, system $(0.1)$ reduces to a system of hyperbolic conservation laws,

$$
\frac{\partial u}{\partial t}+\frac{\partial f(u)}{\partial x}=0 \text {. }
$$

Such systems have been extensively studied. It is known that solutions of $(0.4)$ will not, in general, be smooth $[11,14,20]$, and a certain criterion, the entropy condition, is required to select physically admissible weak solutions $[7,12,13,15]$. Glimm [9] has constructed solutions for $(0.4)$ when the initial data have small total variation. Because the nonlinearity of $f(u)$ and the entropy condition, solutions for (0.4) attain very interesting large-time behavior $[8,10,17,18]$.

Due to the term $g(x, u)$ which represents either geometrical or physical effects, waves for (0.1) propagate in a much more complicated way than do those for (0.4). As a result, system (0.1) exhibits richer nonlinear wave phenomena and is a suitable model for a much wider range of physical phenomena. For instance, the Euler equations with spherical or cylindrical symmetry [5] and the elasticity model capable of describing shearing and flex-turing effects are of the form $(0.1)$ [1]. The one-dimensional model gas flow in a variable area duct is of the form

$$
\begin{gathered}
\frac{\partial \varrho}{\partial t}+\frac{\partial(\varrho u)}{\partial x}=-\frac{a^{\prime}(x)}{a(x)} \varrho u, \\
\frac{\partial(\varrho u)}{\partial t}+\frac{\partial\left(\varrho u^{2}+p\right)}{\partial x}=-\frac{a^{\prime}(x)}{a(x)} \varrho u^{2}, \\
\frac{\partial(\varrho E)}{\partial t}+\frac{\partial(\varrho E u+p u)}{\partial x}=-\frac{a^{\prime}(x)}{a(x)}(\varrho E u+p u),
\end{gathered}
$$

where $a(x)$ is the cross section of the duct, $\varrho, u, p$, and $E$ are, respectively, the density, velocity, pressure and the total energy of the gas. Shock propagation for $(0.5)$ has been studied by suppressing the effects of nonlinear wave interactions [2, $3,24,25]$. The problem of secondary recovery of petroleum, [26], is also related to the present situation because reservoirs in nature have variable thickness. When a moving source term with speed $c$ is applied to the system $(0.4)$ [27], we have

$$
\frac{\partial u}{\partial t}+\frac{\partial f(u)}{\partial x}=h(x-c t)
$$

which is of the form (1) with a change of variable $v(y, t)=u(x, t), y=x-c t$ :

$$
\frac{\partial v}{\partial t}+\frac{\partial(f(u)-c I)}{\partial y}=h(y) .
$$

Our analysis will show that the asymptotic form of the solutions depends mainly on the relative magnitude of the characteristic speeds $\lambda_{i}, i=1,2, \ldots, n$ of $\frac{\partial f}{\partial u}$ and the source speed $c$.

For the physical situations we have in mind, the term $g(x, u)$ does not have a preferred form and does not decay as $t$ goes to infinity. Thus the usual Duhemel principle and the energy method do not seem to be applicable here and the solution may not exist for all time. 
Nevertheless, we will show that when $\lambda_{i}(u(x)), i=1,2, \ldots, n$, are nonzero and when the $L^{1}$ norm of $g(x, u(x))$ and $\frac{\partial g}{\partial u}(x, u(x))$ are small provided that $u(x)$ is uniformly close to the initial data, $u_{0}(x)$, then a global solution of $(0.1)$ and $(0.2)$ exists and tends pointwise to a steady state solution of

$$
\frac{\partial f(u)}{\partial x}=g(x, u)
$$

When each characteristic field is either genuinely nonlinear or linearly degenerate, [12], the solution tends uniformly to the linear supperposition of shock waves, refraction waves, traveling waves and a steady state solution. Moreover these waves are determined by the values of the initial data $u_{0}(x)$ at $x= \pm \infty$. For general systems $(0.1)$ we assume that the initial data $u_{0}(x)$ have small total variation. This assumption is relaxed for the system $(0.5)$ when the gas is polytropic [16]. The main assumptions for the system $(0.5)$ are that the flow at $t=0$ is not close to transonic and that the total variation of the cross section $a(x)$ of the duct is sufficiently small.

We now describe the difference scheme we employ for solving (0.1) and (0.2). Choose an equidistributed sequence $\left\{a_{i}\right\}$, in $(-1,1)$ and mesh length $\Delta x=r, \Delta t=s$ satisfying the Courant-Friedrichs-Lewy condition

$$
r / s \geqq \lambda_{i}(u)
$$

for all eigenvalues $\lambda_{i}$ of (1) and all $u$ under consideration. At $t=0$, the approximate solution $u_{r}(x, 0)$ is a piecewise steady state solution which equals $u_{0}(x)$ at $x=(h+1) r, h$ even,

$$
\begin{aligned}
& u_{r}((h+1) r, 0)=u_{0}((h+1) r), \\
& \frac{d f\left(u_{r}(x, 0)\right)}{d x}=g\left(x, u_{r}(x, 0)\right) \text { for } \quad h r<x<(h+2) r, \quad h \text { even. }
\end{aligned}
$$

By resolving the discontinuities at $x=h r, u_{r}(x, t)$ is defined for $0 \leqq t<s$. Suppose that $u_{r}(x, t)$ has been defined for $0 \leqq t<k s$. Then $u_{r}(x, k s)$ is related to $u_{r}(x, k s-0)$ according to the prechosen equidistributed sequence $\left\{a_{i}\right\}$ in the following way:

$$
\begin{aligned}
u_{r}\left(\left(h+1+a_{k}\right) r, k s\right) & =u_{r}\left(\left(h+1+a_{k}\right) r ; k s-0\right), \\
\frac{d f\left(u_{r}(x, k s)\right)}{d x} & =g\left(x, u_{r}(x, k s)\right), \quad h+k=\text { even, } \quad h r<x<(h+1) r
\end{aligned}
$$

when $g(x, u) \equiv 0, u_{r}(x, k s)$ is a step function and the discontinuities at $x=h r, h+k$ even, are resolved by considering certain related Riemann problems which will be described in Sect. 1, $[12,15,23]$. In this case our scheme is reduced to the Glimm scheme, [9], for solving the conservation law (0.4). For general $g, u_{r}(x, k s)$ has steady state solutions lying adjacent to the discontinuity at $x=h r$. Such discontinuities are resolved in Sect. 2 by perturbing about corresponding Riemann problems and solving a system of nonlinear integro-partial differential equations. To implement the scheme, we have only to resolve the dicontinuities locally in time. It follows from number-theoretic results on equidistributed sequences that 
the Glimm scheme is of less than first order accuracy, [19], thus there is no need to resolve the discontinuities exactly.

Section 3 is devoted to the study of wave interactions for the system (0.1). It is shown that the difference between wave interactions for $(0.1)$ and those for $(0.4),[9$, $18]$ can be dominated by the $L^{1}$ norm of $g(x, u(x))$ and $\frac{\partial g}{\partial u}(x, u(x))$. This enables us to show in Sects. 4 and 6 that our scheme always converges and yields local solution provided that $g(x, u)$ is bounded. Section 5 is devoted to the partition of waves which is needed in the subsequent sections to control the evolution of the speed and the strength of waves. In Sect. 7 we deal with the global existence of the solution. For this we require that the eigenspeeds are nonzero and $g(x, u(x))$ is small in $L^{1}$ so that the total effect of $g$ is finite. This is done by effective uses of the hyperbolicity of the system and the equidistributedness of the sequence $\left\{a_{i}\right\}$. Sections $8-11$ are devoted to the study of the asymptotic behavior of the solution. The nonlinearity of $f(u)$ and the entropy condition force the waves to combine and cancel and so solutions assume a very simple form. In Sect. 8 we study solutions consisting of the linear superposition of elementary waves and a steady state solution. The basic mechanism of wave spreading and combining is described in Sect. 9.

In Sect. 10 we show that the solution tends to a linear superposition of shock waves, rarefaction waves, traveling waves and a steady state solution without rate of convergence. For each genuinely nonlinear characteristic field, the asymptotic form of the solution contains a shock or a rarefaction wave; and, for each linearly degenerate field, a traveling wave. Basic to our arguments is that the total amounts of wave interaction are finite. In Sect. 11 we obtain a stronger asymptotic result when the initial data $u_{0}(x)$ do not depend on $x$ for $|x|>M$, and $g(x, u)$ equals zero for $|x|>M$. We show that the convergence of the solution to shock waves, traveling waves and a steady state solution is of the rate $t^{-3 / 2}$ and the convergence to rarefaction waves is of the rate $t^{-1 / 2}$. For this, we need a detailed analysis of the distribution of wave interactions.

\section{Preliminaries}

We assume that system (0.1) is strictly hyperbolic, i.e., $\partial f / \partial u$ has real and distinct eigenvalues $\lambda_{1}(u)<\lambda_{2}(u)<\ldots<\lambda_{n}(u)$ with right and left eigenvectors $r_{i}(u)$ and $l_{i}(u)$, $i=1,2, \ldots, n$. Although it is not essential for our existence theorem, we will assume for simplicity throughout this paper that each $i$-characteristic field is either genuinely nonlinear (g.nl.) or linearly degenerate (l.dg.) in the sense of Lax [12]

$$
\nabla \lambda_{i}(u) \cdot r_{i}(u) \neq 0 \text { for all } u
$$

or

$$
\nabla \lambda_{i}(u) \cdot r_{i}(u) \equiv 0 \text { for all } u \text {. }
$$

This makes it easier to describe the admissibility of discontinuities and to solve the Riemann problem for system (0.4) [15]. Across any discontinuity $x=x(t)$ of a weak solution $u(x, t)$, the following jump (Rankine-Hugniot) condition is satisfied

$$
\left(u_{+}-u_{-}\right) \sigma\left(u_{-}, u_{+}\right)=f\left(u_{+}\right)-f\left(u_{-}\right)
$$


for some scalar $\sigma\left(u_{-}, u_{+}\right)=x^{\prime}(t)$, the shock speed, where $u=u(x(t) \pm 0, t)$. Under the assumption of genuine-nonlinearity, a discontinuity $\left(u_{-}, u_{+}\right)$is admissible in the sense of Lax [12] if

$$
\lambda_{i}\left(u_{+}\right)<\sigma\left(u_{-}, u_{+}\right)<\lambda_{i}\left(u_{-}\right)
$$

for some $i \in\{1,2, \ldots, n\}$. Such a discontinuity is called an $i$-shock wave.

In the remaining of this section, we study the elementary waves for the conservation laws (0.4). For each genuinely nonlinear field, there are shock waves and rarefaction waves, and for each linearly degenerate field, there are contact discontinuities. A centered $i$-rarefaction wave $\left(u_{0}, u_{1}\right)$ connecting $u_{0}$ on the left and $u_{1}$ the right is a smooth solution of (0.4) which depends only on $x / t$ and takes values on the rarefaction curve $R_{i}\left(u_{0}\right)$, the integral curve of $r_{i}(u)$ through $u_{0}$

$$
\begin{aligned}
\left(u_{0}, u_{1}\right)(x, t) & =u_{0} \quad \text { for } \quad \frac{x}{t} \leqq \lambda_{i}\left(u_{0}\right), \quad\left(u_{0}, u_{1}\right)(x, t)=u_{1}, \quad \text { for } \quad \frac{x}{t} \geqq \lambda_{i}\left(u_{1}\right), \\
\left(u_{0}, u_{1}\right)(x, t) & =v\left(\frac{x}{t}\right) \text { for } \lambda_{i}\left(u_{0}\right) \leqq \frac{x}{t} \leqq \lambda_{i}\left(u_{1}\right), \\
\lambda_{i}\left(u\left(\frac{x}{t}\right)\right) & =\frac{x}{t}, \quad v \in R_{i}\left(u_{0}\right), \\
R_{i}\left(u_{0}\right) & =\left\{u: u \text { is connected to } u_{0} \text { by an integral curve of } r_{i} \text { and } \lambda_{i}(u)\right. \\
& \left.\geqq \lambda_{i}\left(u_{0}\right)\right\}, i \text {-th field g.nl. }
\end{aligned}
$$

A centered $i$-shock wave $\left(u_{0}, u_{1}\right)$ is a discontinuity satisfying conditions $(\mathrm{R}-\mathrm{H})$ and (L):

$$
\begin{aligned}
& \left(u_{0}, u_{1}\right)(x, t)=\left\{\begin{array}{l}
u_{0} \text { for } \quad \frac{x}{t}<\sigma\left(u_{0}, u_{1}\right) \\
u_{1} \quad \text { for } \quad \frac{x}{t}>\sigma\left(u_{0}, u_{1}\right),
\end{array}\right. \\
& u_{1} \in S_{i}\left(u_{0}\right), \\
& S_{i}\left(u_{0}\right)= \\
& \left\{u:\left(u-u_{0}\right) \sigma\left(u_{0}, u\right)=f(u)-f\left(u_{0}\right) \text { for some scalar } \sigma\left(u, u_{0}\right)\right. \\
& \text { and } \left.\lambda_{i}(u)<\sigma\left(u_{0}, u_{1}\right)<\lambda_{i}\left(u_{0}\right), \quad i \text {-th field g.nl. }\right\} .
\end{aligned}
$$

It is easy to see by the implicit function theorem that $S_{i}\left(u_{0}\right)$ is a smooth curve at least for a small neighborhood of $u_{0}$ and is tangent to $R_{i}\left(u_{0}\right)$ at $u_{0}$ up to second derivations $[4,12]$, and

$$
\sigma\left(u_{0}, u\right)=\frac{1}{2}\left(\lambda_{i}\left(u_{0}\right)+\lambda_{i}(u)\right)+0\left(\left|u-u_{0}\right|^{2}\right) .
$$

We set

$$
T_{i}\left(u_{0}\right)=S_{i}\left(u_{0}\right) \cup R_{i}\left(u_{0}\right), \quad i \text {-th field g.nl. }
$$

For 1.dg. $i$-field, we set

$$
T_{i}\left(u_{0}\right)=\left\{u: u \text { is connected to } u_{0} \text { by an integral curve of } r_{i}\right\} .
$$


For $u_{1} \in T_{i}\left(u_{0}\right), i$-th field 1.dg., we have contact discontinuity

$$
\begin{aligned}
& \left(u_{0}, u_{1}\right)=\left\{\begin{array}{lll}
u_{0} & \text { for } & \frac{x}{t}<\sigma\left(u_{0}, u_{1}\right) \\
u_{1} & \text { for } & \frac{x}{t}>\sigma\left(u_{0}, u_{1}\right),
\end{array}\right. \\
& \sigma\left(u_{0}, u_{1}\right)=\lambda_{i}\left(u_{0}\right)=\lambda_{i}\left(u_{1}\right) .
\end{aligned}
$$

Thus for any $i,\left(u_{0}, u_{1}\right)$ is an elementary $i$-wave provided that $u_{1} \in T_{i}\left(u_{0}\right)$.

To solve the Riemann problem $\left(u_{l}, u_{r}\right)$ for $(0.4)$ with

$$
u(x, 0)=\left\{\begin{array}{lll}
u_{l} & \text { for } & x<0 \\
u_{r} & \text { for } & x>0
\end{array}\right.
$$

we find states $u_{i}, i=0,1,2, \ldots, n, u_{0}=u_{l}, u_{n}=u_{r}, u_{i} \in T_{i}\left(u_{i-1}\right)$, so that the solution consists of $i$ waves $\left(u_{i-1}, u_{i}\right)$. The strength $\left(u_{l}, u_{r}\right)_{i}$ of those $i$-waves $\left(u_{i-1}, u_{i}\right)$ is measured by

$$
\begin{aligned}
& \left(u_{l}, u_{r}\right)_{i}=\lambda_{i}\left(u_{i}\right)-\lambda_{i}\left(u_{i-1}\right) \text { for g.nl. } i \text {-field, } \\
& \left(u_{l}, u_{r}\right)_{i}=\tau_{i}\left(u_{i}\right)-\tau_{i}\left(u_{i-1}\right) \text { for g.dg. } i \text {-field, }
\end{aligned}
$$

where $\tau_{i}$ is any nonsingular parameter along $T_{i}$. It is noted that an $i$-shock wave has negative strength while an $i$-rarefaction wave has positive strength. The Riemann problem was first solved for the polytropic gas equation by Riemann [23]. It has been shown by Lax [14] that the Riemann problem $\left(u_{0}, u_{r}\right)$ has a unique solution provided that the system $(0.4)$ is either g.nl. or l.dg. and $u_{l}$ is close to $u_{r}[15]$.

\section{Resolving Discontinuities}

We want to solve the Cauchy problem (0.1) with

$$
u(x, 0)=\left\{\begin{array}{lll}
u_{l}(x) & \text { for } & x<0, \\
u_{r}(x) & \text { for } & x>0
\end{array}\right.
$$

where $u_{l}(x)$ and $u_{r}(x)$ are steady state solutions of $(0.6)$. We will solve the problem for small time and then only approximately. This is done by perturbing about the solution of the corresponding Riemann problem $\left(u_{l}, u_{r}\right), u_{l} \equiv u_{l}(0), u_{r} \equiv u_{r}(0)$, for the conservation laws (0.4). Let $\left(u_{i-1}, u_{i}\right)$ be the $i$-wave in the solution of the Riemann problem $\left(u_{i}, u_{r}\right)$ for $(0.4)$. We denote by $u_{i}(x)$ the steady state solution of $(0.6)$ satisfying $u_{i}(0)=u_{i}$. The approximate solution of $(0.1)$ and (2.1) will consist of steady states $u_{i}(x), i=0,1, \ldots, n, u_{0}(x)=u_{l}(x), u_{n}(x)=u_{r}(x)$, separated by elementarylike $i$-waves. When $\left(u_{i-1}, u_{i}\right)$ is a shock wave or a contact discontinuity, we simply separate $u_{i-1}(x)$ and $u_{i}(x)$ by a discontinuity with speed $\frac{x}{t}=\sigma\left(u_{i-1}, u_{i}\right)$. 
When $\left(u_{i-1}, u_{i}\right)$ is a rarefaction wave, $u_{i-1}(x)$ and $u_{i}(x)$ are separated by certain wave in a region $x_{i-1}(t)<x<x_{i}(t)$. This region and the wave in it are defined as follows: The solution approaches the $i$-rarefaction wave $\left(u_{i-1}, u_{i}\right)$ as $t \rightarrow 0$,

$$
\begin{aligned}
& \left\{\begin{array}{l}
\lim _{t \rightarrow 0} \frac{x_{i-1}(t)}{t}=\lambda_{i}\left(u_{i-1}\right) \equiv \xi_{0} \\
\lim _{t \rightarrow 0} \frac{x_{i}(t)}{t}=\lambda_{i}\left(u_{i}\right) \equiv \xi_{1}
\end{array}\right. \\
& \lim _{t \rightarrow 0} u(x, t)=v(\eta) \quad x_{i-t}(t)<x<x_{i}(t),
\end{aligned}
$$

where $v(\eta) \in R_{i}\left(u_{i-1}\right)$ with $\lambda_{i}(v(\eta))=\eta$. Thus we expect $u(x, t)$ to have a singularity at $t=0$. This singularity is resolved if we use the coordinate of time $t$ and the initial $i$ characterized speed $\xi$ defined as follows:

$$
\begin{aligned}
\frac{\partial x(\xi, t)}{\partial t} & =\lambda_{i}(w(\xi, t)), \quad x(\xi, 0)=0, \quad \xi_{0}<\xi<\xi_{1}, \\
w(\xi, t) & \equiv u(x(\xi, t), t), \quad w(\xi, 0)=v(\xi) \equiv \phi(\xi), \quad \xi_{0}<\xi<\xi_{1} .
\end{aligned}
$$

These are consistent with (2.2) and (2.3) if we set

$$
x_{i-1}(t)=x\left(\xi_{0}, t\right), \quad x_{i}(t)=x\left(\xi_{1}, t\right) .
$$

With the transformation (2.4) and (2.5), the system (0.1) becomes

$$
\begin{aligned}
\frac{\partial x}{\partial \xi} \frac{\partial w}{\partial t}+\left(A-\lambda_{i}\right) \frac{\partial w}{\partial \xi} & =\frac{\partial x}{\partial \xi} g, \xi_{0}<\xi<\xi_{1}, \\
A & \equiv \frac{\partial f}{\partial u} .
\end{aligned}
$$

To determine $w(\xi, t),(2.5)$ and (2.6) have to be supplemented by the boundary condition on $\xi_{0}=\lambda_{i}\left(u_{i-1}\right)$ or $\xi_{1}=\lambda_{i}\left(u_{i}\right)$. For definiteness we set

$$
\begin{aligned}
\frac{\partial x\left(\xi_{0}, t\right)}{\partial t} & =\lambda_{i}\left(u_{i-1}(x)\right), \\
w\left(\xi_{0}, t\right) & \equiv u_{i-1}\left(x\left(\xi_{0}, t\right)\right) \equiv \psi(t) .
\end{aligned}
$$

Since $u_{i-1}(x)$ satisfies $(0.4)$, it follows from (2.7) that

$$
l^{i}(w) \cdot\left(\frac{\partial w}{\partial t}-g(w)\right)=0 \text { for } \xi=\lambda_{i}\left(u_{i-1}\right) .
$$

We now construct $w(\xi, t)$ from $(2.4) \sim(2.7)$. It is noted that $A-\lambda_{i}$ is a singular matrix, and $\frac{\partial x}{\partial \xi}$ vanishes at $t=0$ and involve the derivatives of $w$. In fact, (2.6) is an integro-differential system. Thus the local existence of the solution does not follow from standard result on quasilinear systems [6]. However, we will show that usual iteration scheme yield the solution. 
Multiplying (2.6) on the left with $l_{j}(w)$, we have

$$
\begin{aligned}
D^{j} \delta_{j} & =\frac{\partial x / \partial \xi}{\lambda_{j}-\lambda_{i}} l_{j} \cdot g+D^{j} l_{j} \cdot w \text { for } j \neq i, \quad 1 \leqq j \leqq n, \\
\frac{\partial \delta_{i}}{\partial t} & =l_{j} \cdot g+\frac{\partial l_{j}}{\partial t} \cdot w, \\
\delta_{j} & \equiv l_{j} \cdot w, \quad 1 \leqq j \leqq n, \quad D^{j} \equiv \frac{\partial x / \partial \xi}{\lambda_{j}-\lambda_{i}} \frac{\partial}{\partial t}+\frac{\partial}{\partial \xi}, \quad j \neq i .
\end{aligned}
$$

(2.8) can also be written as

$$
\begin{aligned}
l_{j} \cdot D^{j} w & =\frac{\partial x / \partial \xi}{\lambda_{j}-\lambda_{i}} l_{j} \cdot g, \quad j \neq i, \\
l_{i} \cdot \frac{\partial w}{\partial t} & =l_{i} \cdot g .
\end{aligned}
$$

Integration (2.8) along the integral wave $C_{j}$ of $D^{j}$ (Fig. 1), we have

$$
\begin{aligned}
& \delta_{j}(\eta, s)=\delta_{j}\left(\xi_{0}, \tau\right)+\int_{C_{1}} \frac{\partial x / \partial \xi}{\lambda_{j}-\lambda_{i}} l_{j} g d \xi+\int_{C_{j}} D^{j} l_{j} \cdot w d \xi, \\
& \delta_{i}(\eta, s)=\delta_{i}(\eta, 0)+\int_{0}^{s} l_{i} g d t+\int_{0}^{s} \frac{\partial l_{i}}{\partial t} \cdot w d t .
\end{aligned}
$$

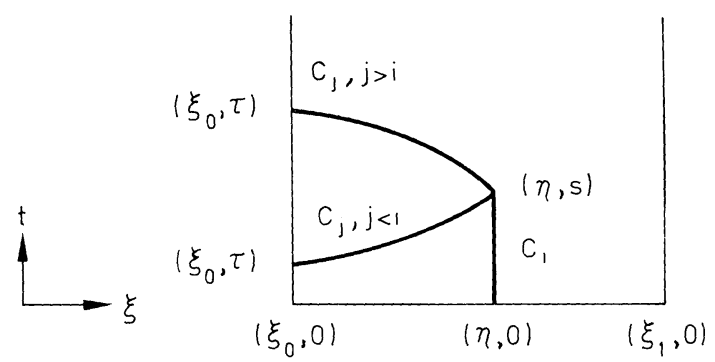

Fig. 1

The curves $C_{j}, j \neq i$, do not intersect $t=0$ provided that

$$
\frac{\partial x}{\partial \xi}=t+O\left(t^{2}\right)
$$

and $t$ is small. In this case the initial-boundary value problem (2.4) and (2.7) is wellposed. For convenience we quote the following elementary facts of calculus: 
Lemma 2.1. Let $\Lambda\left(\beta, \Lambda_{0}\right)$ be the solution of

$$
\begin{aligned}
& \frac{d \Lambda}{d \beta}=\eta(\beta, \Lambda), \quad \Lambda\left(\beta_{0}, \Lambda_{0}\right)=\Lambda_{0} . \\
& \text { Then } \sigma(\beta) \equiv \frac{\partial \Lambda\left(\beta, \Lambda_{0}\right)}{\partial \Lambda_{0}} \text { satisfies } \\
& \sigma(\beta)=\exp \int_{\beta_{0}}^{\beta} \frac{\partial n(\alpha, \Lambda)}{\partial \Lambda} d \alpha, \\
& \frac{d \sigma(\beta)}{d \beta}=\sigma(\beta) \frac{\partial \varphi(\Lambda, \beta)}{\partial \Lambda}, \quad \Lambda \equiv \Lambda\left(\beta, \Lambda_{0}\right) .
\end{aligned}
$$

Lemma 2.2. Suppose that $f(\alpha, \beta)$ and $g(\alpha, \beta)$ are continuously differentiable functions of $\alpha$ and $\beta$. Then

$$
\frac{d}{d \alpha} \int f \frac{\partial g}{\partial \beta} d \beta=\int\left[\frac{\partial f}{\partial \alpha} \frac{\partial g}{\partial \beta}-\frac{\partial f}{\partial \beta} \frac{\partial g}{\partial \alpha}\right] d \beta+f \frac{\partial g}{\partial \alpha} .
$$

By differentiating (2.11), it follows form the lemmas and (2.4) that

$$
\begin{aligned}
& l_{j} \cdot \frac{\partial w}{\partial t}(\eta, s)=l_{j}(\eta, 0) \cdot \psi^{\prime}(\tau) \cdot \frac{d \tau}{d s}+\int_{C_{J}} \frac{d t}{d s}\left\{\frac{\partial \lambda_{i}}{\partial \xi} \frac{l_{j} \cdot g}{\lambda_{j}-\lambda_{i}}\right. \\
& \left.+\frac{\partial x}{\partial \xi} \frac{\partial}{\partial t}\left(\frac{l_{j} \cdot g}{\lambda_{j}-\lambda_{i}}\right)+\frac{\partial w}{\partial t} D^{j} l_{j}-\frac{\partial l_{j}}{\partial t} \cdot D^{j} w\right\} d \xi, \quad j \neq i, \\
& l_{i} \cdot \frac{\partial w}{\partial \xi}(\eta, s)=l_{i}(\eta, 0) \cdot \phi^{\prime}(\eta)+\int_{0}^{s}\left\{\frac{\partial}{\partial \xi}\left(l_{i} \cdot g\right)+\frac{\partial w}{\partial \xi} \frac{\partial l_{i}}{\partial t}-\frac{\partial w}{\partial t} \frac{\partial l_{i}}{\partial \xi}\right\} d t \\
& \frac{d t}{d s}=\exp \left\{-\int_{C_{\imath}(\xi, t)}^{(\eta, s)} \frac{\frac{\partial \lambda_{i}}{\partial \xi}-\frac{\partial x}{\partial \xi} \frac{\partial}{\partial t}\left(\lambda_{j}-\lambda_{i}\right)}{\lambda_{j}-\lambda_{i}} d \xi\right\}, \\
& D^{j} \frac{d t}{d s}=\frac{\frac{\partial \lambda_{i}}{\partial \xi}-\frac{\partial x}{\partial \xi} \frac{\partial}{\partial t}\left(\lambda_{j}-\lambda_{i}\right)}{\lambda_{j}-\lambda_{i}} \frac{d t}{d s} .
\end{aligned}
$$

Theorem 2.3. Suppose that $g(x, u)$ is continuously differentiable. Then for sufficiently small $\Delta \xi=\xi_{1}-\xi_{0}$ and $\Delta t$ the initial-boundary value problem (2.4) (2.7) has an unique smooth solution for $0 \leqq t<\Delta t$ satisfying

$$
\begin{gathered}
\frac{\partial w}{\partial t}=O(1) G_{*}, \\
\frac{\partial w}{\partial \xi}(\xi, t)=\phi^{\prime}(\xi)+O(1) \Delta t G_{*},
\end{gathered}
$$

where $G_{*}=\max _{(x, u)}\{g, \nabla g\}$, the maximum being taken over $(x, u)$ in a neighborhood of $(0, \phi(\xi))$. 
Proof. The solution to $(2.4) \sim(2.7)$ is constructed by the usual double iteration process. We first define the values of $x, \lambda_{j}, l_{j}, g$, and $A$ by setting $w$ equal to a given smooth function $v$ satisfying

$$
v\left(\xi_{0}, t\right)=\psi(t), \quad v(\check{\zeta}, 0)=\phi(\xi),
$$

and denote by

$$
w=T v
$$

the solution of $(2.4) \sim(2.7)$. To show that the operator $T$ exists, we set the function $w$ on the right hand side of $(2.11)$ by a given function and use iteration process, details are omitted. It is noted that on the right-hand sides of $(2.10),(2.11)$ and (2.13), (2.14) only depends on $v$ and its first derivatives. It follows easily that for small $\Delta t$ and $\nabla \xi, T$ is contractive in the max norm of $v, w$ and their first derivatives. This proves the existence of the solution by iterating the operator $T$. In the process, however, we have to check condition (2.12) so that the problem is well-posed. This is done by choosing $v=w_{0}$ to satisfy (2.12) and by induction $w_{n-1}, n=1,2, \ldots$, all satisfy (2.12). Details are omitted. It remains to prove (2.15) and (2.16). For this we require further that $w_{0}$ satisfies (2.15) and (2.16). The existence of such smooth function $w_{0}$ can be shown easily. It follows from (2.10) and (2.13) that each $w_{n}$ satisfies (2.15). As a result of (2.15) and (2.12) we have (2.16). This completes the proof of the theorem. Q.E.D.

Theorem 2.4. Suppose that $\lambda_{j}(u), j=1,2, \ldots, n$, is nonzero $g(x, u), \frac{\partial g(x, u)}{\partial u}$ are continuous for $x$ in a small neighborhood of 0 and $u$ in a small neighborhood of $\phi(\xi)$. Then for sufficiently small $\Delta \xi$ and $\Delta t(2.4) \sim(2.11)$ has a smooth solution $w(x, t)$ for $0 \leqq t<\Delta t$ satisfying

$$
\begin{gathered}
\frac{\partial w}{\partial t}=O(1) G, \\
\frac{\partial w}{\partial \xi}(\xi, t)=\phi^{\prime}(\xi)+O(1) G \Delta t, \\
G=\max _{x} G(x), \quad G(x) \equiv \max _{u} g(x, u)\left\{\frac{\partial g(x, u)}{\partial u}\right\},
\end{gathered}
$$

where the maxima are taken for $x$ near 0 and $u$ near $\phi(\xi)$.

Proof. By chain rule and (2.7), we have

$$
\begin{aligned}
& \frac{\partial g(x, v)}{\partial t}=\frac{\lambda_{i}\left(\lambda_{j}-\lambda_{i}\right)}{\lambda_{j} \partial x / \partial \xi}\left\{D^{j} g+\frac{\partial g}{\lambda v}\left[\frac{\partial x / \partial \xi}{\lambda_{i}} \frac{\partial v}{\partial t}-\frac{\partial v}{\partial \xi}\right]\right\}, \\
& \frac{\partial g(x, v)}{\partial \xi}=\frac{\lambda_{j}-\lambda_{i}}{\lambda_{i}} D^{j} g+\frac{\partial g}{\partial v}\left\{\frac{\lambda_{i}}{\lambda_{j}} \frac{\partial v}{\partial \xi}-\frac{\partial x / \partial \xi}{\partial_{j}} \frac{\partial v}{\partial t}\right\}
\end{aligned}
$$


for any $j \neq i$. In view of these estimates and Lemma 2.2, (2.13) yields

$$
\begin{aligned}
l_{j} \cdot \frac{\partial w}{\partial t}(\eta, s)= & \lambda_{i} l_{j}\left(\xi_{0}, \tau\right) g\left(\xi_{0}, \tau\right) \frac{d x}{d \beta}+\int_{c_{j}} \frac{d t}{d s}\left\{\frac{\partial \lambda_{i}}{\partial \xi} \frac{l_{j} \cdot g}{\lambda_{j}-\lambda_{i}}+\frac{\partial x}{\partial \xi}\right. \\
& \cdot \frac{\partial}{\partial t}\left(\frac{l_{j}}{\lambda_{j}-\lambda_{i}}\right) g+\frac{\partial w}{\partial t} D^{j} l_{j}-\frac{\partial l_{j}}{\partial t} D^{j_{w}}+\frac{l_{j}}{\lambda_{j}} \frac{\partial g}{\partial v} \\
& \cdot\left(\frac{\partial x}{\partial \xi} \frac{\partial v}{\partial t}-\lambda_{i} \frac{\partial v}{\partial \xi}\right)-g \cdot D^{j}\left(\frac{\lambda_{i} l_{j}}{\lambda_{i}}\right) \\
& \left.-\frac{\lambda_{i} l_{j} \cdot g}{\lambda_{j}\left(\lambda_{j}-\lambda_{i}\right)}\left(\frac{\partial \lambda_{i}}{\partial \xi}-\frac{\partial x}{\partial \xi}\left(\frac{\partial \lambda_{j}}{\partial t}-\frac{\partial \lambda_{i}}{\partial t}\right)\right)\right\} \\
& \cdot d \xi+\left.\frac{d t}{d s} \frac{\lambda_{i} l_{j} \cdot g}{\lambda_{j}}\right|_{\left(\xi_{0}, \tau\right)} ^{(\eta, s)} j \neq i, \\
l_{i} \cdot \frac{\partial w}{\partial \xi}(\xi, s)= & l_{i}(\xi, 0) \cdot \phi^{\prime}(\xi)+\int_{0}^{s}\left\{\frac{\partial w}{\partial \xi} \frac{\partial l_{i}}{\partial t}-\frac{\partial w}{\partial t} \frac{\partial l_{i}}{\partial \xi}\right. \\
& +\frac{\partial l_{i}}{\partial \xi} \cdot g+l_{i} \cdot \frac{\partial g}{\partial v}\left(\frac{\lambda_{i}}{\lambda_{j}} \frac{\partial v}{\partial \xi}-\frac{\partial x}{\partial \xi} \frac{\partial v}{\partial t} \frac{1}{\lambda_{j}}\right) \\
& \left.-D^{j}\left(\frac{\lambda_{j}-\lambda_{i}}{\lambda_{j}} l^{i}\right) \cdot g\right\} d t+\left.\frac{\lambda_{j}-\lambda_{i}}{\lambda_{j}} l_{i} \cdot g\right|_{(\xi, 0)} ^{(\xi, s)},
\end{aligned}
$$

where we have used the fact that $u_{i-1}(x)$ is a steady state solution and $\xi=\xi_{0}$ is an $i$ characteristic curve for (0.1) so that

$$
\phi^{\prime}(\tau)=\lambda_{i} g\left(\xi_{0}, \tau\right) .
$$

Finally the theorem is proved by the iteration techniques used in the proof of Theorem 2.3 along with estimates (2.10) and (2.19). Q.E.D.

We are now ready to resolve the discontinuity (2.1) for the system $(0.1)$.

Theorem 2.5. Suppose that $g(x, u)$ and $\frac{\partial g(x, u)}{\partial u}$ are continuous. Then for sufficiently small $\Delta t$ and $\Delta u \equiv\left|u_{l}-u_{r}\right|, u_{l}=u_{l}(0), u_{r} \equiv u_{r}(0)$, the initial value problem (0.1) and (2.1) has an approximate solution $u(x, t)$ for $0 \leqq t<\Delta t$ with the following properties. Let $\left(u_{i-1}, u_{i}\right), i=1,2, \ldots, n$, be the $i$-waves in the solution of the Riemann problem $\left(u_{l}, u_{r}\right)$ for $(0.4)$ and $u_{i}(x), i=1,2, \ldots, n$, are the steady state solutions of $(0.6)$ with $u_{i}(0)=u_{i}$. Then

i) when $\left(u_{i-1}, u_{i}\right)$ is a contact discontinuity or a shock wave, $u(x, t)$ contains a discontinuity along $x=\sigma\left(u_{i-1}, u_{i}\right)$ t separating $u_{i-1}(x)$ and $u_{t}(x)$ which almost satisfies the Rankine-Hugoniot condition:

$$
\begin{aligned}
{[u(x-0, t)-u(x+0, t)] \sigma\left(u_{i-1}, u_{i}\right)=} & f(u(x-0, t)-f(u(x+0, t)) \\
& +O(1) G\left|u_{i-1}-u_{i}\right| t,
\end{aligned}
$$

ii) when $\left(u_{i-1}, u_{i}\right)$ is a rarefaction wave, $\left(u_{i-1}, u_{i}\right) \equiv v(x / t)$ for $\lambda_{i}\left(u_{i-1}\right)<x / t$ $<\hat{i}_{i}\left(u_{i}\right)$, then $u(x, t)$ contains a wedge-shaped wave in $x_{i-1}(t)<x<x_{i}(t)$ separating $u_{i-1}(x)$ and $u_{i}(x)$ such that $u(x, t)$ is an exact solution of $(0.1)$ for $x_{i-1}(t) \leqq x \leqq x_{i}(t)$ with possible discontinuity along $x_{i}(t)$, where $x=x_{i-1}(t)$ and $x=x_{i}(t)$ are 
$i$-characteristic curves of $u(x, t)$ with initial speed $\lambda_{i}\left(u_{i-1}\right)$ and $\lambda_{i}\left(u_{i}\right)$ respectively at $t=0$. Moreover, along any $i$-characteristic curves $x=x_{1}(t)$ and $x=x_{2}(t)$ in $x_{i-1}(t)$ $<x_{1}(t) \leqq x_{2}(t)<x_{i}(t)$, with initial speed $\lambda_{i}^{1}$ and $\lambda_{1}^{2}$ respectively, $u(x, t)$ satisfies

$$
\begin{aligned}
& \left|u\left(x^{j}(t), t\right)-v\left(\lambda_{i}^{j}\right)\right|=O(1) G t, \\
& \left|u\left(x^{1}(t), t\right)-u\left(x^{2}(t), t\right)\right|=v\left(\lambda_{i}^{1}\right)-v\left(\lambda_{i}^{2}\right)+O(1) G t\left|v\left(\lambda_{i}^{1}\right)-v\left(\lambda_{i}^{2}\right)\right|, \\
& x^{j}(t)=\lambda_{i}^{j} t+O(1) G t^{2}, \quad j=1,2 .
\end{aligned}
$$

Finally, the discontinuity of $u(x, t)$ along $x_{i}(t)$ satisfies

$$
\left|u\left(x_{i}(T)-0, t\right)-u\left(x_{i}(t)+0, t\right)\right|=O(1) G t\left|u_{t-1}-u_{i}\right|,
$$

were $G \equiv \max _{x, u}\left\{|g|,\left|g_{u}\right|\right\}$ for u over a neighborhood of $u_{l}(0)$ and $u_{r}(0)$ and $x$ near zero.

Proof. Except for the strength of discontinuities in $u(x, t)$, the above theorems follow from Theorem 2.4. When $\left(u_{i-1}, u_{i}\right)$ is a shock wave or a contact discontinuity, then it follows from Lemma 2.1 that the corresponding discontinuity in $u(x, t)$ satisfies

$$
u(x-0, t)-u(x+0, t)=u_{i-1}+u_{i}+O(1)\left|u_{i-1}-u_{i}\right| t .
$$

Since $\left(u_{i-1}, u_{i}\right)$ satisfies the Rankine-Hugoniot condition, the above estimate shows that $(u(x-0, t), u(x+0, t))$ almost satisfies the Renkine-Hugoniot condition as stated in the theorem. Suppose that $\left(u_{i-1}, u_{i}\right)$ is a rarefaction wave. By our construction,

$$
u\left(x_{i-1}(t), t\right)=u_{i-1}\left(x_{i-1}(t)\right) \text { and } u\left(x_{i}(t)+0, t\right)=u_{i}\left(x_{i}(t)\right) .
$$

We have from Lemma 2.1 that

$$
u_{i-1}\left(x_{i}(t)\right)-u_{i}\left(x_{i}(t)\right)=u_{i-1}-u_{i}+O(1) G t\left|u_{i-1}-u_{i}\right| .
$$

Since $x_{i}(t)-x_{i-1}(t)=O(1) t\left|u_{i-1}-u_{i}\right|$ as follows from (2.12), we have

$$
u_{i-1}\left(x_{i}(t)\right)=u_{i-1}\left(x_{i-1}(t)\right)+O(1) G t\left|u_{i-1}-u_{i}\right| .
$$

We have from (2.16) that

$$
u\left(x_{i-1}(t), t\right)-u\left(x_{i}(t)-0, t\right)=u_{i-1}-u_{i}+O(1) G t\left|u_{1-1}-u_{i}\right| .
$$

Finally the above estimates imply that

$$
u\left(x_{i}(t)-0, t\right)-u\left(x_{i}(t)+0, t\right)=O(1) G t\left|u_{i-1}-u_{i}\right|
$$

as asserted. Q.E.D.

The following corollary is an easy consequence of the above theorem:

Corollary 2.6. The approximate solution $u(x, t)$ in the above theorem satisfies

$$
\begin{aligned}
\int_{0}^{\Delta t} & \left.\int_{-\infty}^{\infty} u \frac{\partial \phi}{\partial t}+f(u) \frac{\partial \phi}{\partial x}-g(x, u) \phi\right) d x d t+\int_{-\infty}^{\infty} u(x, 0) \phi(x, 0) d x \\
= & \int_{-\infty}^{\infty}(u(x, 0) \phi(x, 0)-u(x, \Delta t) \phi(x, \Delta t)) d x+O(1) G\left|u_{r}-u_{l}\right|(\Delta t)^{2}
\end{aligned}
$$

for any test function $\phi$ smooth and with compact support in $t \geqq 0$. 


\section{Local Interactions}

Given three steady state solutions $u_{l}(x), u_{m}(x)$ and $u_{r}(x)$ of $(0.6)$, we want to compare the solution of the initial value problem $(0.1)$,

$$
u(x,)=\left\{\begin{array}{lcc}
u_{l}(x) & \text { for } & x<-\theta \\
u_{m}(x) & \text { for } & -\theta<x<\theta, \\
u_{r}(x) & \text { for } & x>\theta,
\end{array}\right.
$$

with the solution of $(0.1)$ and

$$
u(x, 0)=\left\{\begin{array}{lll}
u_{l}(x) & \text { for } & x<0 \\
u_{r}(x) & \text { for } & x>0
\end{array}\right.
$$

for some positive constant $\theta$. We set

$$
\begin{cases}v_{-} \equiv u_{l}(-\theta), & v_{+} \equiv u_{m}(-\theta) \\ w_{-} \equiv u_{m}(\theta), & w_{+} \equiv u_{r}(\theta) \\ u_{-} \equiv u_{l}(0), & u_{+} \equiv u_{r}(0)\end{cases}
$$

Denote by $\left(\delta_{1-1}, \delta_{i}\right), \delta=u, v, w$, the $i$-wave, $i=1,2, \ldots, n$, in the solution of the Riemann problems $\left(\delta_{-}, \delta_{+}\right)$for conservation laws $(0.4)$ and set

$$
\alpha_{i} \equiv\left(v_{-}, v_{+}\right)_{i}, \quad \beta_{i} \equiv\left(w_{-}, w_{+}\right)_{i}, \quad \gamma_{i} \equiv\left(u_{-}, u_{+}\right)_{i} \quad i=1,2, \ldots, n .
$$

Theorem 3.1. Suppose that $u_{l}(x), u_{m}(x)$, and $u_{r}(x)$ are close to a constant. Then for some bounded $O(1)$ depending only on the system $(0.1)$

$$
\gamma_{i}=\alpha_{i}+\beta_{i}+O(1) Q_{0}\left(v_{ \pm}, w_{ \pm}\right)+O(1) Q_{1}\left(v_{ \pm}, w_{ \pm}\right) .
$$

Here $Q_{0}=Q_{0}^{s}+Q_{0}^{d}$ measures the potential nonlinear wave interaction between $\left\{\left(v_{i-1}\right.\right.$, $\left.\left.v_{i}\right), i=1,2, \ldots, n\right\}$ and $\left\{\left(w_{i-1}, w_{i}\right), i=1,2, \ldots, n\right\}$, and $Q_{1}$ measures the effect of the inhomogeneous term $g(x, u)$ :

$$
\begin{aligned}
& Q_{0}^{s}\left(v_{ \pm}, w_{ \pm}\right)=\sum\left\{Q_{i}^{s}: i \in\{1,2, \ldots, n\}, i \text {-field is g.nl. }\right\}, \\
& Q_{i}^{s}= \begin{cases}0 & \text { if } \alpha_{i} \geqq 0, \quad \beta_{i} \geqq 0, \\
\left|\alpha_{i}\right|^{3}-\left|\alpha_{i}+\beta_{i}\right|^{3} & \text { if }-\alpha_{i} \geqq \beta_{i} \geqq 0, \\
\left|\alpha_{i}\right|^{3} & \text { if } \beta_{i} \geqq-\alpha_{i}>0, \\
\left|\beta_{i}\right|^{3} & \text { if } \alpha_{i} \geqq-\beta_{i}>0, \\
\left|\beta_{i}\right|^{3}-\left|\alpha_{i}+\beta_{i}\right|^{3} & \text { if }-\beta_{i} \geqq \alpha_{i} \geqq 0, \\
8\left|\alpha_{i} \beta_{i}\right|\left|\alpha_{i}+\beta_{i}\right| & \text { if } \alpha_{i}<0, \quad \beta_{i}<0 .\end{cases} \\
& Q_{0}^{d}=\sum_{i>j}\left|\alpha_{i} \beta_{j}\right|, \\
& Q_{1}=G \theta \sum_{i=1}^{n}\left(\left|\alpha_{i}\right|+\left|\beta_{i}\right|\right),
\end{aligned}
$$

where $G$ is the maximum of $g(x, u)$ and $\frac{\partial g}{\partial u}(x, u)$ for $-\theta>x>\theta$ and $u$ in a small neighborhood of $v_{ \pm}$and $w_{ \pm}$. 
Proof. We denote by $u_{m} \equiv u_{m}(0)$. It follows from elementary theory of ordinary differential equations (cf. Lemma 2.1) that

$$
\begin{aligned}
& v_{+}-v_{-}=u_{m}-u_{-}+O(1) G \theta\left|v_{+}-v_{-}\right| \\
& w_{+}-w_{-}=u_{+}-u_{m}+O(1) G \theta\left|w_{+}-w_{-}\right| .
\end{aligned}
$$

Since the solution of the Riemann problem depends continuously on its data, we have

$$
\begin{aligned}
& \alpha_{i}=\left(u_{-}, u_{m}\right)_{i}+O(1) G \theta\left|v_{+}-v_{-}\right|, \quad i=1,2, \ldots, n, \\
& \beta_{i}=\left(u_{m}, u_{+}\right)_{i}+O(1) G \theta\left|w_{+}-w_{-}\right|, \quad i=1,2, \ldots, n .
\end{aligned}
$$

The wave interaction for conservation laws $(0.4)$ has been studied $[9,16,17]$ and it has been shown (Theorem 3.3 of [16] and Theorem 2.2 of [17]) that our theorem holds when $g \equiv 0$. Thus we have from the above estimates that

$$
\gamma_{i}=\alpha_{i}+\beta_{i}+O(1) Q_{0}\left(u_{-}, u_{m}, u_{+}\right)+O(1) G \theta\left(\left|v_{+}-v_{-}\right|+\left|w_{+}-w_{-}\right|\right) .
$$

It is clear that $Q_{0}$ depends continuously on its arguments and thus we have from (3.17) and the above estimate that

$$
\gamma_{i}=\alpha_{i}+\beta_{i}+O(1) Q_{0}\left(v_{ \pm}, w_{ \pm}\right)+O(1) G \theta\left(\left|v_{+}-v_{-}\right|-\left|w_{+}-w_{-}\right|\right) .
$$

The theorem follows easily from the above estimates. Q.E.D.

\section{Local Estimates}

The purpose of this section is to obtain estimates on total variation of approximate solutions locally in time. This is done by introducing a nonlinear functional $F_{*}(J ; T)$ defined on any $I$-curve $J$. An $I$-curve is a spacelike curve connecting points of the form $\left(\left(m+a_{n}\right) \gamma, n s\right), m+n$ odd, $J_{2}$ is called an immediate successor of $J_{1}$ if they pass through same mesh points except one and $J_{2}$ lies toward larger time than $J_{1}$. The region sandwiched by such two $I$-curves is called a diamond $\Delta$. An $i$ wave issued from $(m r, n s), m+n$ even, crosses $J$ if it is contained in the solution of the Riemann problem corresponding to the discontinuity of the approximate solution at $(m r, n s)$ and there is no mesh point between $J$ and $(m r, n s)$. For a given positive $T$, we set

$$
\begin{aligned}
F_{*}(J ; T)= & L(J)+K\left[Q_{0}(J)+Q_{*}(J ; T)\right], \\
L(J)= & \sum_{J}\{|\alpha|: \alpha \text { is the strength of any } i \text {-wave, } i=1,2, \ldots, n, \text { cross- } \\
& \text { ing } J\}, \\
Q_{0}(J)= & Q_{0}^{d}(J)+Q_{0}^{s}(J), \\
Q_{0}^{d}(J)= & \sum\{|\alpha \beta|: \alpha, \beta \text { crossing } J, \alpha \text { is the strength of any } i \text {-wave lying } \\
& \text { toward the left of a } j \text {-wave with strength } \beta, i>j\}, \\
Q_{0}^{s}(J)= & \sum\left\{Q_{i}^{s}(J), i \text {-field is g.nl. }\right\}, \\
Q_{i}^{s}(J)= & \sum\left\{|\alpha|^{3}: \alpha \text { is the strength of any } i \text {-shock wave crossing } J\right\} \\
& +8 \sum\{|\alpha+\beta||\alpha \beta|: \alpha, \beta \text { are strengths of any } i \text {-shock waves } \\
& \text { crossing } J\}, \text { or }
\end{aligned}
$$




$$
\begin{aligned}
Q_{i}^{s}(J)= & \sum_{\text {and not both of them are rarefaction waves }\},}\{|\alpha+\beta|\{\alpha \beta \mid: \alpha, \beta \text { are strengths of any } i \text {-waves crossing } J \\
Q_{*}(J ; T)= & \sum_{J}\left\{G_{0}(T-k s)|\alpha|: \quad \alpha \text { is the strength of any } i\right. \text {-wave, } \\
& i=1,2, \ldots, n, \text { crossing } J \text { and issued from }(h r, k s)\}, \\
G_{0}= & \sup \left\{|g|,\left|g_{u}\right|: x \in R, u \in N\left(u_{0}\right)\right\}
\end{aligned}
$$

where $K$ is some large constant to be determined later. In the definition of $Q_{*}(J ; T)$ above, we assume that the $I$-curve $J$ is contained in the strip $0 \leqq t \leqq T$.

Theorem 4.1. Let $T$ be a fixed positive constant. Suppose that the total variation $T V$ of the initial data $(0.2)$ and the $L_{\infty}(x)$ norm $G_{0}$ and the $L_{1}(x)$ norm $G_{1}$ of

$$
G(x)=\max _{u}\left\{|g(x, u)|+\left|\frac{\partial g(x, u)}{\partial u}\right|: u \text { in a neighborhood of } u_{0}(x)\right\},
$$

are small. Then there exists a positive constant $K$ such that

$$
F_{*}\left(J_{2} ; T\right) \leqq F_{*}\left(J_{1} ; T\right)
$$

for any $I$-waves $J_{1}$ and $J_{2}$ in the strip $0 \leqq t \leqq T, J_{2}$ an immediate successor of $J_{1}$.

Proof. The theorem is proved by induction. Assuming that $F\left(J_{1}\right)$ is small, we will show that (4.3) holds and thus $F\left(J_{2}\right)$ is also small. That $F(0), 0$ the $I$-curve in the strip $0 \leqq t \leqq s$, is small follows easily from the definition (4.1) and that $T V+G_{0}+G_{1}$ is small.

Let $\Delta$ be the diamond between $J_{1}$ and $J_{2}$. Assume for simplicity that the waves entering $\Delta$ and leaving $\Delta$ correspond to the solutions of the Riemann problems $\left(\delta_{-}, \delta_{+}\right), \delta=u, v, w$, of $(3.1) \sim(3.4)$. Here we have set $\theta$ of (3.1) to be equal to the mesh length $\Delta x=r$. We denote $Q_{0}\left(v_{ \pm}, w_{ \pm}\right) \equiv Q_{0}(\Delta)$, and $Q_{1}(\Delta)=Q_{1}\left(v_{ \pm}, w_{ \pm}\right)$. Naturally, in the definition of $Q_{1}\left(v_{ \pm}, w_{ \pm}\right)$we set $G=\max _{x}\left\{g, \frac{\partial g}{\partial u}\right\}$ for $(m-1) \gamma<x<(m+1) \gamma$ if $\Delta$ is centered at $(m r, n s)$. It follows from Theorem 3.1 that

$$
L\left(J_{2}\right) \leqq L\left(J_{1}\right)+O(1) Q_{0}(\Delta)+O(1) Q_{1}(\Delta) .
$$

Since the $i$-waves $\left(u_{\imath-1}, u_{i}\right), i=1,2, \ldots, n$, do not interact among themselves, we have from (4.4), (4.1) $3,(4.1)_{4}$ that

$$
Q_{0}\left(J_{2}\right) \leqq Q_{0}(J)+L\left(J_{1}\right)\left[O(1) Q_{0}(\Delta)+O(1) Q_{1}(\Delta)\right]-Q_{0}(\Delta) .
$$

Since $J_{2}$ lies toward a larger time than $J_{1}$, the definition $(4.1)_{b}$ and Theorem 3.1 imply that

$$
\begin{aligned}
Q_{*}\left(J_{2} ; T\right) & \leqq Q_{*}\left(J_{1} ; T\right)+G_{0}\left[O(1) Q_{0}(\Delta)+O(1) Q_{1}(\Delta)\right]-s G_{0} \sum_{i=1}^{n}\left(\left|\alpha_{i}\right|+\left|\beta_{\imath}\right|\right) \\
& \leqq Q_{*}\left(J_{1} ; T\right)+G_{0}\left[O(1) Q_{0}(\Delta)+O(1) Q_{1}(\Delta)\right]-\frac{s}{r} Q_{1}(\Delta)
\end{aligned}
$$


Finally it follows from $(4.4) \sim(4.6)$ that

$$
\begin{aligned}
F_{*}\left(J_{2} ; T\right)-F_{*}\left(J_{1} ; T\right) \leqq & Q_{0}(\Delta)\left[-K+O(1)+K O(1)\left(Q_{0}(\Delta)+Q_{1}(\Delta)\right)\right] \\
& +Q_{1}(\Delta)\left[-K \frac{S}{r}+O(1)+K\left(O(1)\left(Q_{0}(\Delta)+Q_{1}(\Delta)\right)\right] ;\right.
\end{aligned}
$$

Since $\frac{s}{r}$ is bounded away from zero, it follows from the above estimate that (4.3) holds when $F_{*}\left(J_{1}\right)$ is sufficiently small by choosing $K$ sufficiently large. Q.E.D.

For a region $\Lambda$ composed of diamonds, we set

$$
Q_{0}(\Lambda)=\sum_{\Delta \in \Lambda} Q_{0}(\Delta), \quad Q_{1}(\Lambda)=\sum_{\Delta \in \Lambda} Q_{1}(\Delta)
$$

Assuming that the waves entering $\Delta$ are $\left\{\alpha_{i}\right\}$ and $\left\{\beta_{i}\right\}, i=1,2, \ldots, n$, we set

$$
\begin{aligned}
& C_{i}(\Delta)=\frac{1}{2}\left\{\left|\alpha_{i}\right|+\left|\beta_{i}\right|-\left|\alpha_{i}+\beta_{i}\right|\right\}, \\
& C_{i}(\Lambda)=\sum_{\Delta \in A} C_{i}(\Delta) .
\end{aligned}
$$

The following lemma is an easy consequence of Theorem 4.1 and its proof.

Lemma 4.2. Suppose that the hypothesos of Theorem 4.1 hold and $A$ is between $J_{1}$ and $J_{2}$ in $0 \leqq t \leqq T, J_{2}$ lies toward larger time than $J_{1}$. Then

$$
\begin{aligned}
Q_{0}(\Lambda)+Q_{1}(\Lambda) & \leqq 2\left[Q_{0}\left(J_{1}\right)-Q_{0}\left(J_{2}\right)+Q_{*}\left(J_{1} ; T\right)-Q_{*}\left(J_{2} ; T\right)\right], \\
C(\Lambda) & \leqq L(J)+O(1)\left[Q_{0}(\Lambda)+Q_{1}(\Lambda)\right] .
\end{aligned}
$$

\section{Partition of Waves}

Suppose that the discontinuity of the approximate solution at $(m r, n s)$, $m+n=$ even, is resolved into elementary $i$-waves $\left(u_{i-1}(x), u_{i}(x)\right), i=1,2, \ldots, n$.

Locally these waves are approximate solutions of $(0.1)$ as described in Theorem 2.5. The corresponding Riemann problem for $(0.4)$ is then resolved into $i$-waves $\left(u_{i-1}, u_{i}\right), u_{i-1} \equiv u_{i-1}(m r), u_{i} \equiv u_{i}(m r)$. We will partition the elementary waves in the following way [19]. If $\left(u_{i-1}, u_{i}\right)$ is an $i$-shock wave or an $i$-contact discontinuity for $(0.6)$ with speed $\sigma\left(u_{i-1}, u_{i}\right)$, then we choose vectors $y_{0}, y_{1}, \ldots, y_{l}, y_{0}=u_{i-1}, y_{l}=u_{i}$, $y_{h} \in S_{i}\left(u_{i-1}\right), h=1,2, \ldots, l$, and set

$$
\begin{aligned}
& v_{i}^{h}(m, n)=y_{h}-y_{h-1}, \\
& \lambda_{i}^{h}(m, n)=\sigma\left(u_{i-1}, u_{i}\right) .
\end{aligned}
$$

We let $\tilde{y}_{h}(x)$ be the steady state solution of $(0.6)$ with $\tilde{y}_{h}(m r)=y_{h}$, and set $y_{h}(t)=\tilde{y}_{h}(x)$, for $x-m r=\sigma\left(u_{i-1}, u_{i}\right)(t-n s)$ and $v_{h}(m, n ; t)=y_{h}(t)-y_{h-1}(t), \lambda_{i}^{h}(m, n ; t)=\lambda_{i}^{h}(m, n)$. If $\left(u_{i-1}, u_{i}\right)$ is an $i$-rarefaction wave, we choose vectors $y_{0}, y_{1}, \ldots, y_{l}, y_{0}=u_{i-1}, y_{l}=u_{i}$, $y_{h} \in R_{i}\left(u_{i-1}\right), h=1,2, \ldots, l$ and set

$$
\begin{aligned}
& v_{i}^{h}(m, n)=y_{h}-y_{h-1}, \\
& \lambda_{i}^{h}(m, n)=\lambda_{i}\left(y_{h-1}\right) .
\end{aligned}
$$


Moreover, we set $y_{h}(t)$ to be the value of the $i$-wave $\left(u_{i-1}(x), u_{i}(x)\right)$ along $i$ characteristic curve $x=x(t)$ with initial speed $\lambda_{i}\left(y_{h-1}\right)$ at $(m r, n s)$ [cf. Theorem $2.5(\mathrm{ii})]$ and $v_{i}^{h}(m, n ; t)=y_{h}(t)-y_{h-1}(t), \lambda_{i}^{h}(m, n ; t)=\lambda_{i}\left(y_{h-1}(t)\right)$. Let $x_{i-1}(t)$ and $x_{i}(t)$ be the boundary of the elementary wave $\left(u_{i-1}(x), u_{i}(x)\right)$ as described in (ii), Theorem 2.5. Note that there is a discontiuity along $x=x_{i}(t)$ with strength $Q(\Delta)$. For this reason, we set $v_{i}^{l}(m, n)$ to be the strength of this discontinuity. We always make the partition fine enough so that

$$
\left|\lambda_{i}^{h}(m, n ; t)-\lambda_{i}^{h-1}(m, n ; t)\right| \leqq \varepsilon, \quad h=1,2, \ldots, l, n s \leqq t<(n+1) s,
$$

for a prescribed small positive constant $\varepsilon$. To make sure that $v_{i}^{h}(m, n ; t)$ is not partitioned further at $t=(m+1) \mathrm{s}$ because of the approximation procedure $(0.7)$, we further require that

$$
a_{m+1} \notin\left(\lambda_{i}^{h-1}(m, n ; t), \lambda_{i}^{h}(m, n ; t)\right), \quad i=1,2, \ldots, n, \quad h=1,2, \ldots, l .
$$

Lemma 5.1. Let $\Lambda$ be the horizontal strip $0 \leqq t \leqq T$. There exists a partition of elementary waves $\left\{v_{i}^{h}(m, n ; t) ; \lambda_{i}^{h}(m, n ; t)\right\}$ which satisfies $(5.1) \sim(5.4)$, and, moreover, $\left\{v_{i}^{h}(m, n ; t) ; \lambda_{i}^{h}(m, n ; t)\right\}$ is a disjoint union of $\left\{\tilde{v}_{i}^{h}(m, n ; t), \tilde{\lambda}_{i}^{h}(m, n ; t)\right\}$ and $\left\{\tilde{\tilde{v}}_{i}^{h}(m, n ; t)\right.$, $\left.\tilde{\lambda}_{i}^{h}(m, n ; t)\right\}$, so that for some bound $O(1)$ depending only on $(0.1)$

$$
\begin{aligned}
& \sum_{i, h, m} \max _{t}\left\{\left\|\tilde{\tilde{v}}_{i}^{h}(m, n ; t)\right\|:(m r, n s) \in \Lambda, m+n=\text { even, } n s \leqq t \leqq(n+1) s\right\} \\
& \quad=O(1)\left[Q_{0}(\Lambda)+Q_{1}(\Lambda)+C(\Lambda)\right]
\end{aligned}
$$

and there is a one-to-one correspondence between $\left\{\tilde{v}_{i}^{h}(m, n), \quad \tilde{\lambda}_{i}^{h}(m, n)\right\}$ and $\left\{\tilde{v}_{i}^{h}(m, 0), \tilde{\lambda}_{i}^{h}(m, 0)\right\}$ :

$\left.\left(\tilde{v}_{i}^{h}(m(n, h, k), n), \tilde{\lambda}_{i}^{h} m(n, h, k), n\right)\right) \leftrightarrow\left(\tilde{v}_{i}^{h}(m, 0), \tilde{\lambda}_{i}^{h}(m, 0)\right)$

such that

$$
\begin{aligned}
& \sum_{i, h, m} \max _{\tau, t}\left\{\left\|\tilde{v}_{i}^{h}(m, 0 ; \tau)-\tilde{v}_{i}^{h}(m(n, h, i) n ; t)\right\|: 0 \leqq \tau \leqq s, n s \leqq t \leqq(n+1) s\right\} \\
& \quad=O(1)\left[Q_{0}(\Lambda)+Q_{*}(\Lambda)\right], \\
& \sum_{i, h, m} \max _{\tau, t, n}\left\{\left\|\tilde{v}_{i}^{h}(m, 0, \tau)\right\|\left|\tilde{\lambda}_{i}^{h}(m(n, h, k) n ; t)-\tilde{\lambda}_{i}^{h}(m, 0, \tau)\right|: 0 \leqq \tau \leqq s,\right. \\
& \quad n s \leqq t \leqq(n+1) s ; 0 \leqq n s \leqq T\}=O(1)\left[Q_{0}(\Lambda)+Q_{*}(\Lambda)\right], \\
& m(n, h, i)=m(n-1, h, i) \mp 1 \quad \text { if } \quad a_{n} r \geqq \tilde{\lambda}_{i}^{h}(m(n-1, h, i), n-1) s, \\
& m(0, h, i)=m .
\end{aligned}
$$

Proof. It follows from Theorem 2.5 and Lemma 2.1 that

$$
\sum_{h} v_{i}^{h}(m, n ; t)=\sum_{h} v_{i}^{h}(m, n)+O(1) Q(\Delta),
$$

$\Delta$ being the diamond centering at $(m r, n s)$. Thus estimates $(5.5) \sim(5.7)$ hold provided that $(5.5)^{\prime} \sim(5.7)^{\prime}$, obtained from $(5.5) \sim(5.7)$ by replacing $\tilde{v}_{i}^{h}(m, n ; t)$ with $\tilde{v}_{i}^{h}(m, n)$ etc., are valid. $(5.5)^{\prime} \sim(5.7)^{\prime}$ are proved by induction. Supposed that elementary waves crossing $u$ below $J$, have been partitioned and satisfy $(5.5)^{\prime}$ $\sim(5.7)^{\prime}$. Let $J_{2}$ be any immediate successor of $J_{1}$ and $\Delta$ the diamond between $J_{1}$ 
and $J_{2}$. Since waves entering $\Delta$ have been partitioned, we may partition waves leaving $\Delta$ accordingly. However, according to Theorem 3.1, this procedure can be carried out except for an error of the order $Q_{0}(\Delta)+Q_{1}(\Delta)+C(\Delta)$. This is remedied by partitioning the waves finer and designate the error terms to be in $\left\{\tilde{u}_{i}^{h}(m, n)\right\}$, still keeping (5.5) valid. For an $i$-rarefaction wave, $v_{l}^{i}(m, n)$ is always classified as $\tilde{\tilde{v}}_{i}^{l}(m, n)$ which has strength $Q_{1}(\Delta)$. Whenever necessary, we make the partition finer so as to satisfy (5.4). It is noted that when $(5.4)^{\prime} \sim(5.7)^{\prime}$ hold for a partition, they hold also for finer partitions. Details are omitted. Q.E.D.

\section{Local Existence}

Theorem 6.1. Under the same hypothesis as in Theorem 4.1, the initial value problem $(0.1)$ and $(0.2)$ has a solution $u(x, t)$ for $0 \leqq t \leqq T$ satisfying

$$
\text { Total variation }\{u(x, t):-\infty<x<\infty\}=O(1)\left[T . V .+G_{1}\right] 0 \leqq t \leqq T,
$$

for some $O(1)$ depending only on the system (0.1) and $T$.

Proof. That the approximate solutions tend to a limiting function $u(x, t)$ as the mesh lengths $r, s$ tend to zero follows from a compactness argument based on the Helly's theorem and from the estimate on the total variation of the approximate solutions in Theorem 4.1 [9]. Estimate (6.1) follows from (4.3) since it is clear from the scheme that $F(0)=O(1)\left[T V+G_{1}\right]$. To show that the limiting function $u(x, t)$ is a weak solution of $(0.1)$ and $(0.2)$ we require that the sequence $\left\{a_{n}\right\}$ be equidistributed [19]. We need to show that $u(x, t)$ satisfies $(0.3)$. For approximate solutions $u_{r}(x, t)$ we have

$$
\begin{aligned}
& \iint_{t \geqq 0}\left(u_{r} \frac{\partial \phi}{\partial t}+f\left(u_{r}\right) \frac{\partial \phi}{\partial x}-g\left(x, u_{r}\right) \phi\right) d x d t+\int_{-\infty}^{\infty} u_{0}(x) \phi(x, 0) d x \\
& =\sum_{n=0}^{\infty}\left(E_{n}^{1}(r)+E_{n}^{2}(r)\right), \\
& E_{n}^{1}(r)=\int_{-\infty}^{\infty}\left[u_{r}(x, n s+0)-u_{r}(x, n s-0)\right] \phi(x, n s) d x, \\
& E_{n}^{2}(r)=\int_{n s}^{(n+1) s} \int_{-\infty}^{\infty}\left[u_{r} \frac{\partial \phi}{\partial t}+f\left(u_{1}\right) \frac{\partial \phi}{\partial x}-g\left(x, u_{r}\right) \phi\right] d x d t .
\end{aligned}
$$

In the present situation, we require that $\phi$ vanishes for $t \geqq T$. It follows from Lemma 2.6 that $u_{r}(x, t)$ is almost an exact solution for $n s<t<(n-1) s$ :

$$
\sum_{n=0}^{\infty} E_{n}^{2}(r)=O(1) s Q_{1}(\Lambda)
$$

which tends to zero as $r, s$ tend to zero. It remains to show that $E_{n}^{1}(r)$ tends to zero as $r, s$ tend to zero. This follows easily from the equidistributedness of $\left\{a_{n}\right\}$ and the lemma provided that we let $\varepsilon$ of (5.3) goes to zero and that $Q_{0}(\Lambda)+Q_{*}(\Lambda)$ $+C(\Lambda) \equiv 0$. The effects of nonlinear interactions $Q_{0}(\Lambda)+Q_{*}(\Lambda)$ and cancellations 
$C(\Lambda)$ are minimized by taking advantage of the fact that the subsequence $\left\{a_{n} \mid q N\right.$ $\leqq n<P N\}$ of $\left\{a_{n}\right\}$ is also equidistributed for any fixed $p>q$, as $N \rightarrow \infty$ and apply Lemma 5.1 to each regions $(p-1) N s \leqq t \leqq P N s, p=1,2, \ldots, N$ being large and $N s$ small. Details are omitted [19]. Q.E.D.

It is noted that in the proof of the local estimate Theorem 4.1, we do not have to assume that $T V, G$, and $G_{1}$, are small. Instead, due to the hyperbolicity of the system (0.1) we need only to assume that the initial data have small local total variation and $T G$ is small in order to obtain local estimates and thus the local existence of the solution for $0 \leqq t \leqq T$. The classical iteration technique for constructing solutions local in time has been applied only for smooth initial data and yields only smooth local solutions [6].

\section{Global Existence}

In this section we will use the assumption that $\lambda_{i}(u) \neq 0, i=1,2, \ldots, n$, for all $u$ near the initial data in an essential way to obtain global existence of the solution. Our first step is to improve on estimates (4.10) and (4.11). For given $T=N s, s$ the mesh length in time direction, we denote by $J_{T}$ the $I$-curve in $0 \leqq t \leqq T$, and lying toward larger time than any $I$-curve in $0 \leqq t \leqq T$. The region between the $I$-curve 0 in $0 \leqq t \leqq s$ and $J_{T}$ is denoted by $\Lambda_{T}$. We set

$$
G_{m, n}=\max _{x}\{G(x):(m-1) r \leqq x \leqq(m+1) r\},
$$

where $G(x)$ was given in (4.2). Suppose the elementary waves entering a diamond $\Delta$ have strength $\left\{\alpha_{i}\right\}$ and $\left\{\beta_{i}\right\}, i=1,2, \ldots, n$, and $\Delta$ is centered at ( $\left.m r, n s\right)$. Then we set

$$
\begin{aligned}
Q_{1}(\Delta) & =r G_{m, n} \sum_{i}\left(\left|\alpha_{i}\right|+\left(\beta_{i} \mid\right),\right. \\
Q_{1}(\Delta) & =\sum_{\Delta \in \Lambda} Q_{1}(\Delta), \\
Q(\Lambda) & =Q_{0}(\Lambda)+Q_{1}(\Lambda) .
\end{aligned}
$$

Using the notations in Sects. 4 and 5, we define

$$
\begin{aligned}
F_{1}(J ; T)= & L(J)+K Q_{0}(J)+K \sum_{\substack{m, h, i \\
N \geqq n \geqq n_{0}}}\left\{\| \tilde{v}_{i}^{h}(m, 0) r G_{m(n, h, i), n}: \tilde{v}_{i}^{h}\left(m_{n_{0}}, n_{0}\right) \text { crossing } J\right\} \\
& +K T G \sum_{\Delta}\left\{Q_{0}(\Delta)+Q_{*}(\Delta): \Delta \text { lies between } J \text { and } J_{T}\right\},
\end{aligned}
$$

for any $J$ in $\Lambda_{T}$. In the following theorems we always assume that the assumptions in Theorem 4.1 hold.

Lemma 7.1. There exists a positive constant $K$ such that for any $J_{2}$ and $J_{1}$ in $\Lambda_{T}, J_{2}$ an immediate successor of $J_{1}$,

$$
\begin{aligned}
F_{1}\left(J_{2} ; T\right) \leqq F_{1}\left(J_{1} ; T\right) & \\
Q_{0}\left(\Lambda_{T}\right) \leqq & 2\left(Q_{0}(0)-Q_{0}\left(J_{T}\right)+2 \sum_{\substack{m, h, i \\
N \geqq n \geqq 0}} r G_{m(n, h, i), n}\left\|\tilde{v}_{i}^{h}(m, 0)\right\|\right. \\
& +2 T G\left(Q_{0}\left(\Lambda_{T}\right)+Q_{*}\left(\Lambda_{T}\right)\right) .
\end{aligned}
$$


Proof. Suppose that $J_{1}$ and $J_{2}$ sandwhich a diamond $\Delta$ centering at $(m r, n s)$. We have from (7.1), (5.6) and Theorem 3.1 that

$$
\begin{aligned}
F_{1}\left(J_{2} ; T\right)-F_{1}\left(J_{1} ; T\right) \leqq & L\left(J_{2}\right)-L\left(J_{1}\right)+K\left(Q_{0}\left(J_{2}\right)-Q_{0}\left(J_{1}\right)\right) \\
& -K\left[Q_{1}(\Delta)+r G O(1)\left(Q_{0}(\Delta)+Q_{1}(\Delta)\right)\right] \\
& -K T G\left(Q_{0}(\Delta)+Q_{1}(\Delta)\right), \\
L\left(J_{2}\right)-L\left(J_{1}\right) \leqq & O(1)\left(Q_{0}(\Delta)+Q_{1}(\Delta)\right), \\
Q_{0}\left(J_{2}\right)-Q_{0}\left(J_{1}\right) \leqq & O(1)\left[Q_{0}(\Delta)+Q_{1}(\Delta)\right] L\left(J_{1}\right)-Q_{0}(\Delta) .
\end{aligned}
$$

The estimate (7.3) follows from the above estimates and the induction hypothesis that $F_{1}\left(J_{1}\right)$ is small. (7.4) is proved in a similar way. Q.E.D.

Lemma 7.2. Suppose that $\left\{a_{n}\right\}$ is equidistributed in $(-1,1)$. Then

$$
\begin{aligned}
& \left\|\tilde{v}_{i}^{h}(m, 0)\right\| \sum_{\geqq n \geqq 0}\left\{r G_{m, n}: m=m(n, h, i)\right\} \\
& =\frac{r}{s}\left\|v_{i}^{h}(m, 0)\right\|\left|\int_{m r}^{m(N, h, i) r} G(x) d x\right|+O(1) T \delta+O(1) T\left(Q_{0}(\Lambda)+(\Lambda)\right),
\end{aligned}
$$

where $\delta=\delta(N)$ tends to zero as s tends to zero or, equivalently, as $N$ tends to infinity. Proof. As $s$ tends to zero, it follows from the equidistributedness of $\left\{a_{n}\right\}$ and (5.8), that

$$
m(n, h, i)=m+\lambda_{i}^{h}(m, 0) n s+O(1) n s\left[\delta+\sum_{k=0}^{n}\left|\lambda_{i}^{h}(m, 0)-\lambda_{i}^{h}(m(k, h, i), k)\right|\right],
$$

where $\delta$ measures equidistributedness of $\left\{a_{n}\right\}$ in $(-1,1)$ and tends to zero as $s$ tends to zero. The estimate (7.5) now follows easily from the above estimate and (5.7). Q.E.D.

For any $I$-curve $J$, we set

$$
\begin{aligned}
Q(J)= & Q_{0}(J)+Q_{1}(J), \\
Q_{1}(J)= & \sum_{J}\left\{|\alpha| \int_{m r}^{\infty} G(x) d x: \alpha \text { is the strength of any } i\right. \text {-wave issued from } \\
& (m r, n s), \text { crossing } J \text { and with positive speed }\} \\
& +\sum_{J}\left\{|\alpha| \int_{-\infty}^{m r} G(x) d x: \alpha \text { is the strength of any } i\right. \text {-wave issued } \\
& \text { from }(m x, n s), \text { crossing } J \text { and with negative speed }\} . \\
F(J)= & L(J)+K Q(J) .
\end{aligned}
$$

Lemma 7.3. Suppose that $T$ is sufficiently small. Then

$$
\begin{aligned}
Q_{1}\left(\Lambda_{T}\right) \leqq & 2\left[Q_{1}(0)-Q_{1}\left(J_{T}\right)\right]+O(1) \delta T \\
& +O(1) T G\left[Q_{0}\left(\Lambda_{T}\right)+Q_{*}\left(\Lambda_{T}\right)\right] .
\end{aligned}
$$


Proof. It follows from the definition (7.1) of $Q_{1}\left(\Lambda_{T}\right)$ and Lemma 5.1 that

$$
\begin{aligned}
Q_{1}\left(\Lambda_{T}\right) \leqq & \sum_{i, h}\left\|v_{i}^{\sim h}(m, 0)\right\|_{N \geqq n \geqq 0}\left\{\gamma G_{m, n}: m=m(n, h, i)\right\} \\
& +O(1) \gamma G\left[Q_{0}\left(\Lambda_{T}\right)+Q_{*}\left(\Lambda_{T}\right)\right]
\end{aligned}
$$

which is less than the right hand side of (7.8) by Lemma 7.2. Q.E.D.

Lemma 7.4. Suppose that $T$ is sufficiently small. Then

$$
\begin{aligned}
Q\left(\Lambda_{T}\right) \leqq & 3\left[Q(0)-Q\left(J_{T}\right)\right]+O(1) \delta T \\
& +O(1) T G\left[Q_{0}\left(\Lambda_{T}\right)+Q_{*}\left(\Lambda_{T}\right)\right] .
\end{aligned}
$$

Proof. Since $Q=Q_{0}+Q_{1}$ and we have stimate (7.8) for $Q_{1}$, we have only to obtain analogous estimate for $Q_{0}$. This estimate follows easily from (7.4) and (7.5). Q.E.D.

Lemma 7.5. Suppose that $T$ is sufficiently small and $K$ is sufficiently large. Then

$$
F\left(J_{T}\right)-F(0) \leqq O(1) T[\delta+T G] .
$$

Proof. We have from Theorem 3.1 and (7.1) that

$$
L\left(J_{T}\right)-L(O) \leqq O(1) Q(\Lambda)
$$

and thus we have from (7.7) and Lemma 7.4 that

$$
\begin{aligned}
F\left(J_{T}\right)-F(O) & \leqq O(1) Q(\Lambda)+K\left[Q\left(F_{T}\right)-Q(O)\right] \\
& \leqq(K-3 O(1))\left[Q\left(J_{T}\right)-Q(O)\right] .
\end{aligned}
$$

For sufficiently large $K$, we have $K-3 O(1)>0$. From Lemma 7.4 we see that

$$
Q\left(J_{T}\right)-Q(O) \leqq \frac{1}{3}\left[O(1) \delta T+O(1) T G\left(Q_{0}\left(\Lambda_{T}\right)+Q_{*}\left(\Lambda_{T}\right)\right]\right.
$$

which is $O(1) T(\delta+T G]$. Q.E.D.

Theorem 7.6. Let $T_{0}$ be any positive constant and $J_{T_{0}}$ be the I-wave lying toward larger time than any I-wave in $0 \leqq t \leqq T_{0}, 0$ the $I$-curve in $0 \leqq t \leqq s$, and $\Lambda$ the region between $J_{T_{0}}$ and 0 . Then for sufficiently small $T V$, the total variation of the initial data $u_{0}(x)$, and $G_{1}$, the $L_{1}(x)$ norm of $G(x) \equiv \max \left\{g(x, u), \frac{\partial g(x, u)}{\partial u}\right\}$,

$$
\begin{aligned}
& F\left(J_{T_{0}}\right) \leqq F(O)+\varepsilon_{1}, \\
& Q(\Lambda) \leqq 3\left(Q(O)-Q\left(J_{T_{0}}\right)\right)+\varepsilon_{2},
\end{aligned}
$$

for some errors $\varepsilon_{1}$ and $\varepsilon_{2}$ which tend to zero as $r$, s tend to zero.

Proof. We apply Lemmas 7.4 and 7.5 repeatedly to the regions $\Lambda_{p}:(p-1) T \leqq t \leqq p T, 1 \leqq p \leqq M$, where $T=\frac{T_{0}}{M}$. When $M$ tends to infinity, $T$ tends to 
zero and from (7.10) we have

$$
\begin{aligned}
F\left(J_{T_{0}}\right)-F(O) & =\sum_{p=1}^{M} F\left(J_{T_{p}}\right)-F\left(J_{T_{p-1}}\right) \\
& \leqq M[O(1) T G(\delta+T) \\
& \leqq O(1) T_{0} G(\delta+T)
\end{aligned}
$$

which tends to zero as $T$ tends to zero. (7.12) is proved similarly. Q.E.D.

The following global existence theorem follows from Theorem 7.6 and the results in Sect. 6.

Theorem 7.7. Suppose that $T V$, the total variation of the initial data $u_{0}(x)$, and $G_{1}$, the $L_{1}(x)$ of $G(x) \equiv \max \left[|g(x, u)|+\left|\frac{\partial g(x, u)}{\partial u}\right|\right], u$ in a small neighborhood of $U_{0}(x)$, $\{-\infty<x<\infty\}$ are sufficiently small, and $\lambda_{i}(u)$ is nonzero for $u$ in a small neighborhood of $u_{0}(x),-\infty<x<\infty$. Then the initial value problem $(0.1)$ and $(0.2)$ has a solution $u(x, t)$ for $t \geqq 0$ satisfying

total variation $\{u(x, t):-\infty<x<\infty\}=O(1)\left(T V+G_{1}\right)$,

for some bounds $O(1)$ depend only on the system (0.1).

Remarks. It is clear that slight modification of our techniques yield same existence results when $g$ and $\frac{\partial g}{\partial u}$, instead of being continuous, are piecewise continuous in $x$. Thus our results apply to system (0.5) when the cross section $a(x)$ of the duct is piecewise continuously differentiable. Moreover, if the gas is polytropic then our techniques combined with those in Liu [16] yield existence theorem for $(0.5)$ when the initial data may not have small total variation (see also $[21,22]$ ).

\section{Simple Solutions}

We will study simple solutions of $(0.1)$, solutions which do not produce interactions and cancellations, and in the following sections show that these solutions are the asymptotic forms of solutions of general initial value problem $(0.1)$ and $(0.2)$. Since the initial data $u_{0}(x)$ have bounded total variation, we may set

$$
u_{l}=u_{0}(-\infty), \quad u_{r}=u_{0}(+\infty) .
$$

The eigenvalues are nonzero:

$$
\lambda_{1}(u)<\ldots<\lambda_{p}(u)<0<\lambda_{p+1}(u)<\ldots<\lambda_{n}(u) \quad p \in\{0,1,2, \ldots, n\}
$$

for all $u$ in a small neighborhood of $u_{0}(x)$. Recall that the $i$-curve $T_{i}\left(u_{0}\right)$ through $u_{0}$ (see Sect.2) consists of points which can be connected to $u_{0}$ on the left by an $i$ elementary wave for the conservation law $(0.4)$. 
Theorem 8.1. Suppose that $u_{l}$ and $u_{r}$ are close, $g(x, u)$ and $\frac{\partial g}{\partial u}(x, u)$ have small $L_{1}(x)$ norm, $G$, and (8.2) holds for all $u$ in a small neighborhood of $u_{l}$ and $u_{r}$. Then there exists a unique set of points $\left\{u_{0}, u_{1}, \ldots, u_{n}, u_{-}, u_{+}\right\}$in a small neighborhood of $u_{l}$ and $u_{r}$ with the following properties:

$$
\begin{aligned}
& u_{0}=u_{l}, \quad u_{n}=u_{r} \quad u_{p}=u_{-}, \\
& u_{i} \in T_{i}\left(u_{i-1}\right), \quad i=1,2, \ldots, p, p+2, \ldots, n, \\
& u_{p+1} \in T_{p}\left(u_{+}\right),
\end{aligned}
$$

and $u_{-}$is related to $u_{+}$by a steady state solution $u(x)$ of $(0.6)$ :

$$
u( \pm \infty)=u_{ \pm} .
$$

Proof. We first note that for a given $u_{-}, u_{+}$is determined uniquely and $u(x)$ stays near $u_{-}$and $u_{+}$because $G_{1}$ is small and $\lambda_{i}(u), i=1,2, \ldots, n$, are nonzero. Thus if we let $u_{-}$moves along $T_{i}\left(u_{*}\right), i=1,2, \ldots, p, u_{*}$ any point in a neighborhood of $u_{l}$ and $u_{r}$, then $u_{+}$moves along a smooth curve $\tilde{T}_{i}\left(u_{*}\right)$. Moreover, Lemma 2.1 implies that $\tilde{T}_{i}\left(u_{l}\right), i=1,2, \ldots, p$, has a tangent

$$
\tilde{r}_{i}\left(u_{*}\right)=r_{i}\left(u_{*}\right)+O(1) G_{1}
$$

at $u_{+}$, because $T_{i}\left(u_{*}\right)$ has a tangent $r_{i}\left(u_{*}\right)$ at $u_{*}$. For $\left(\varrho_{1}, \varrho_{2}, \ldots, \varrho_{n}\right)$ in a small neighborhood of zero, we define

$$
H\left(\varrho_{1}, \varrho_{2}, \ldots, \varrho_{n}\right)=u
$$

whenever there exist $u_{i}, i=0,1,2, \ldots, n, u_{-}, u_{+}$, such that $(8.3)_{2}$ and $(8.3)_{3}$ hold, $u_{0}$ $=u_{l}, u_{p}=u_{-}$and $u_{n}=u$ and

$$
\tau_{i}\left(u_{i}\right)-\tau_{i}\left(u_{i-1}\right)=\varrho_{i}, \quad i=1,2, \ldots, n .
$$

Here $\tau_{i}, i=1,2, \ldots, n$ is any fixed parameter along $T_{i}$. It is clear that $u_{l}$ and $u_{*} \equiv H(0,0, \ldots, 0)$ are related by a steady state solution. To complete the proof, we need to show that $H$ is one-to-one and onto in a neighborhood of the origin. By implicit function theorem this the case if $H$ has a nonsingular Jacobian at the origin. We have

$$
\frac{\partial H(0,0, \ldots, 0)}{\partial \varrho}=\left(\gamma_{1}\left(u_{l}\right), \ldots, \gamma_{p}\left(u_{l}\right), \tilde{\gamma}_{p+1}\left(u_{l}\right), \ldots, \gamma_{n}\left(u_{l}\right)\right) .
$$

It follows from (8.4) and the strict hyperbolicity of the system (0.1) that this matrix is nonsingular for small $G_{1}$. This completes the proof of the theorem. Q.E.D.

\section{Global Interactions}

Given a region $\Lambda$ composed of diamonds, we denote by $E_{i}^{+}(\Lambda)$ and $E_{i}^{-}(\Lambda)$, respectively, the amount of $i$-shock waves entering $\Lambda$. The amounts of $i$-waves leaving $\Lambda$ are denoted by $L_{i}^{ \pm}(\Lambda)$. We have from Theorem 3.1 that

$$
L_{i}^{ \pm}(\Lambda)=E_{i}^{ \pm}(\Lambda) \mp C_{i}(\Lambda)+O(1) Q(\Lambda) .
$$


Let $J$ be an $I$-curve which approaches time $=t$ as $r, s$ tend to zero and denote by $Q(t)$ the limit of $Q(J)$ as $r, s$ tend to zero. Similarly, the terms $Q(\Lambda), C(\Lambda)$ etc. also refer to their limits as $r, s$ tend to zero. The total amount of $i$-shock and $i$ rarefaction waves, respectively, at time $t$ is denoted by $X_{i}^{-}(T)$ and $X_{i}^{+}(t)$. We have from Theorem 7.6 that

$$
\begin{aligned}
Q(\Lambda) & \leqq 3 Q(t), \\
\Lambda & \equiv\{(x, s): s \geqq t\} .
\end{aligned}
$$

An $i$-generalized characteristic curve $\chi_{i}$ is a Lipschitz continuous curve traveling either with $i$-shock or $i$-characteristic speed. The strength of $\chi_{i}$, Str. $\chi_{i}$, is the amount of $i$-shock wave, $i$-th field g.nl., contained in $\chi_{i}$. An $i$-wave may cross $\chi_{i}$ only due to interactions.

Lemma 9.1. Given any time $T \geqq 0$ and an arbitrary small $\varepsilon>0$, choose a positive $M$ large enough so that total variation $\left\{\{u(x, t):|x| \geqq M\}+\int_{-\infty}^{-M} G(x) d x+\int_{M}^{\infty} G(x) d x \leqq \varepsilon\right.$.

Through $(-M, T)$ and $(M, T)$, respectively, we draw $\chi_{i}^{1}$ and $\chi_{i}^{2}, i=1,2, \ldots, n$. Because of the strict hyperbolicity of (0.1), these characteristics do not intersect after time $T^{*}>T$, and, because $\lambda_{i}(u)$ are nonzero for all $u$ under consideration, these characteristics leave the region $|x| \leqq M$ after time $T^{*}$, We have

$$
\begin{aligned}
\tilde{X}_{i}\left(T^{*}\right) & =O(1)(\varepsilon+Q(\Lambda)), \\
Q_{0}^{d}\left(T^{*}\right)+Q_{1}\left(T^{*}\right) & =O(1)\left(T V+G_{1}\right)(\varepsilon+Q(\Lambda)), \\
\Lambda & \equiv\{(X, t): t \geqq T\},
\end{aligned}
$$

where $\tilde{X}_{i}\left(T^{*}\right)$ is the total amount of $i$-waves not in the region $\Lambda_{i}$ between $\chi_{i}^{1}$ and $\chi_{i}^{2}$ over all $t \geqq T^{*}$.

Proof. Since $i$-waves not in $\Lambda_{i}$ are those produced by interactions or those related to the $i$-waves crossing time $t=T$ at $|x|>M$ [cf. (9.1)]. The total amount of such waves are $O(1)(\varepsilon+Q(\Lambda))$. This proves (9.3). Note that $i$-waves between $\chi_{i}^{1}$ and $\chi_{i}^{2}$, do not interact with $j$-waves between $\chi_{j}^{1}$ and $\chi_{j}^{2}$ after time $T^{*}$ and thus do not contribute to $Q_{0}^{d}\left(T^{*}\right)$. Since $\chi_{i}^{1}$ and $\chi_{i}^{2}$ leave $|x| \leqq M$ after time $T^{*}$, the contribution of $i$-waves between $\chi_{i}^{1}$ and $\chi_{i}^{2}$ is at most $\varepsilon T V$. The above two facts and the estimate (9.3) yield (9.4). Q.E.D.

Lemma 9.2. Under the same hypothesis as in Lemma 9.1, let $Y_{i}^{ \pm}(t)$ be the amount of $i$-rarefaction and $i$-shock waves between $\chi_{i}^{1}$ and $\chi_{i}^{2}$ at time $t \geqq T^{*}$, and $D_{i}(t)$ be the distance between $\chi_{i}^{1}$ and $\chi_{i}^{2}$ at time $t$ then for g.nl. $i$-field and $t \geqq T^{*}$,

$$
\begin{aligned}
Y_{i}(t) & \leqq \frac{D_{i}(t)}{t-T^{*}}+O(1)[\varepsilon+Q(\Lambda)], \\
\Lambda & \equiv\{(x, t): t \geqq T\} .
\end{aligned}
$$

Proof. Let $u_{ \pm}^{j}(s), j=1,2$, be the one-sided limits of $u(x, t)$ along $\chi_{i}^{j}$ at time $s$, and Str. $\chi_{i}^{j}(s), j=1,2$, the strength of $\chi_{i}^{j}$ at time $s$. We have from the jump condition:

$$
\frac{d D_{i}(s)}{d s}=\sigma_{i}\left(u_{-}^{2}(s), u_{+}^{2}(s)\right)-\sigma_{i}\left(u_{-}^{1}(s), u_{+}^{1}(s)\right)
$$


which lies between $\lambda_{-}^{2}(s)-\lambda_{+}^{1}(s)$ and $\lambda_{+}^{2}(s)-\lambda_{-}^{1}(s), \lambda_{+}^{1}(s) \equiv \lambda_{i}\left(u_{+}^{1}(s)\right)$ etc., because the $i$-field is g.nl. and the entropy condition (L) holds. Therefore we have from the mean value theorem that there exists $\theta=\theta(s), 0<\theta<1$, such that

$$
\begin{aligned}
\frac{d D_{i}(s)}{d s} & =\theta\left(\lambda_{-}^{2}(s)-\lambda_{+}^{1}(s)\right)+(1-\theta)\left(\lambda_{+}^{2}(s)-\lambda_{-}^{1}(s)\right) \\
& =\lambda_{-}^{2}(s)-\lambda_{+}^{1}(s)+(1-\theta)\left(\operatorname{Str} \cdot \chi_{i}^{1}(s)+\operatorname{Str} . \chi_{1}^{2}(s)\right) .
\end{aligned}
$$

We recall that the strength of $i$-waves is measured by the jump in $\lambda_{i}$, and the amount of $j$-waves, $j \equiv i$, between $\chi_{i}^{1}$ and $\chi_{i}^{2}$ is

$$
\sum_{j=i} \tilde{X}_{j}(s)=O(1)(\varepsilon+Q(\Lambda))
$$

Thus we have

$$
\begin{aligned}
\frac{d D_{i}(s)}{d s}= & Y_{i}^{+}(s)+Y_{i}^{-}(s)+O(1)(\varepsilon+Q(\Lambda)) \\
& +(1-\theta)\left(\text { Str. } \chi_{i}^{1}(s)+\text { Str. } \chi_{i}^{2}(s)\right) .
\end{aligned}
$$

Applying (9.1) to the region between $\chi_{i}^{1}, \chi_{i}^{2}$, time $=T$ and time $=s$, we obtain

$$
\begin{aligned}
& Y_{i}^{+}(s) \geqq Y_{i}^{+}(t)-O(1) Q(\Lambda), \quad T^{*} \leqq s \leqq t, \\
& Y_{i}^{-}(s) \geqq Y_{i}^{-}\left(T^{*}\right)-O(1) Q(\Lambda), \quad T^{*} \leqq s \leqq t .
\end{aligned}
$$

Integrating (9.8) from $T^{*}$ to $t$ and using the above estimates we have

$$
Y_{i}^{+}(T) \leqq \frac{D_{i}(t)}{t-T^{*}}+O(1)(\varepsilon+Q(\Lambda))+O(1)|\max \mathrm{Str} .|
$$

where max Str. denotes the maximum strength of $\chi_{i}^{1}$ and $\chi_{i}^{2}$ between time $T^{*}$ and $t$. By applying the above arguments to regions with predominant $i$-rarefaction waves (cf. [10], Sect. 8), the above estimate can be improved to yield (9.5). Details are omitted. Q.E.D.

\section{Weak Asymptotic Behavior}

In this section we will show that solutions tend to their asymptotic form with no rate of convergence. Our sole assumption is that the initial data have small total variation $T V$ and $G(x) \equiv \max _{u}\left\{g(x, u), \frac{\partial g(x, u)}{\partial u}\right\}$ has small $L_{1}(x)$ norm $G_{1}$, and the characteristic speed $\lambda_{i}(u)$ are nonzero for all $u$ under consideration. We will employ the notations $u_{l}, u_{r}, u_{0}, u_{1}, \ldots, u_{n}, u_{-}$and $u_{+}$defined by $(8.1) \sim(8.3)$. The primary $i$-region $\Omega_{i}, i=1,2, \ldots, n$, and $\Omega_{*}$ are defined as follows :

$$
\begin{aligned}
\Omega_{0} & =\left\{(x, t): x<\mu_{0} t\right\}, \\
\Omega_{i} & =\left\{(x, t): \mu_{i-1} t<x<\mu_{i} t\right\}, \quad i=1,2, \ldots, p, p+2, \ldots, n, \\
\Omega_{p+1} & =\left\{(x, t): \mu_{*} t<x<\mu_{p+1} t\right\}, \\
\Omega_{*} & =\left\{(x, t): \mu_{p} t<x<\mu_{*} t\right\},
\end{aligned}
$$


where $\mu_{i}, i=1,2, \ldots, n$, and $\mu_{*}$ are constants separating $\lambda_{i}, i=1,2, \ldots, n$, and zero, i.e., for some positive constant $\delta$ and for all $u$ under consideration,

$$
\begin{aligned}
\mu_{0} & \leqq \lambda_{i}(u)-\delta, \\
\lambda_{i}(u)+\delta & \leqq \mu_{i} \leqq \lambda_{i+1}(u)-\delta, \quad i=0,1, \ldots, p-1,-+1, \ldots, n, \\
\mu_{p} & \leqq-\delta \\
\delta & \leqq \mu_{*} \leqq \lambda_{p+1}(u)-\delta
\end{aligned}
$$

Theorem 10.1. Suppose that the total variation of the initial data $u_{0}(x)$ and the $L_{1}(x)$ norm of $G(x)=\max _{u}\left\{g(x, u), \frac{g(x, u)}{u}: u\right.$ in a small neighborhood of $\left.u_{0}(x)\right\}$ are small and (8.2) holds. Then the solution $u(x, t)$ of $(0.1)$ and $(0.2)$ tends to elementary waves $\left(u_{i-1}, u_{i}\right)$, $i=1,2, \ldots, n$, and a steady state $\left(u_{-}, u_{+}\right)$defined by $(8.1) \sim(8.3)$ as $t \rightarrow \infty$. More precisely,

i) The amount of $i$-waves, $i=1,2, \ldots, n$, not in $\Omega_{i}$ tends to zero.

ii) In the region $\Omega_{*}, u(x, t)$ approaches uniformly and in total variation norm to the steady state solution of (0.6) relating $u_{-}$on the left to $u_{+}$on the right.

iii) If the $i$-field is linearly degenerate, then $u(x, t) / \Omega_{i}$ tends to a traveling wave which connects $u_{i-1}$ on the left to $u_{i}$ on the right and takes values along $T_{i}\left(u_{-}\right)$.

iv) If the $i$-field is genuinely nonlinear and $\left(u_{i-1}, u_{i}\right)$ is an i-rarefaction wave for $(0.4)$, then $u(x, t) / \Omega_{i}$ tends to $\left(u_{i-1}, u_{i}\right)$ pointwise uniformly, and the amount of $i$-shock wave in $u(x, t) / \Omega_{i}$ tends to zero.

v) If the $i$-field is genuinely nonlinear and $\left(u_{i-1}, u_{i}\right)$ is an $i$-shock wave for $(0.4)$, then there emerges an $i$-shock wave in $u(x, t) / \Omega_{i}$ which tends to $\left(u_{i-1}, u_{i}\right)$ and outside the $i$-shock wave $u(x, t) / \Omega_{i}$ tends to zero in total variation norm.

Proof. Since $Q(\Lambda)$ is finite for any $\Lambda$ as follows from (9.2), we may take $t$ sufficiently large so that $Q(\Lambda)$ is $(9.5)$ less than any prescribed number $\tilde{\varepsilon}$. It is noted that $\Lambda_{i}$ in Lemma 9.1 is contained eventually in $\Omega_{i}$. Thus i) of the theorem follows from (9.3) by letting $\varepsilon$ and $\tilde{\varepsilon}$ approach zero. As a consequence of i), and the set-up of our scheme, $(0.7), u / \Omega_{*}$ tends to a steady state solution, and, because $\int_{\Omega_{\imath}} G(x) d x$ tends to zero as $t \rightarrow \infty, u / \Omega_{i}$, $i$-field l.dg., tends to a traveling wave. The left and right limits of the steady state solution and the traveling wave are yet to be determined. Another consequence of $i$ ) is the following: The amount of waves crossing $\frac{x}{t}=\mu_{i}$, $i=0,1, \ldots, n$, and $\frac{x}{t}=\mu_{*}$ approaches zero as $t$ tends to zero. As a result, the following limits exist:

$$
\begin{aligned}
v_{i} & \equiv \lim _{t \rightarrow \infty} u\left(\mu_{i} t, t\right), \quad i=0,1, \ldots, n, \quad v_{p} \equiv v_{-}, \\
v_{+} & \equiv \lim _{t \rightarrow \infty} u\left(\mu_{*} t, t\right) .
\end{aligned}
$$

It remains to show that $v_{i}=u_{i}, i=0,1,2, \ldots, n, u_{ \pm}=u_{ \pm}$, and in $\Omega_{v}, i$-field g.nl., the solution tends to either shock or rarefaction wave. 
Suppose that the $i$-field is g.nl. and $\lambda_{i}\left(v_{i}\right)<\lambda_{i}\left(v_{i-1}\right)$. Choose $T$ and $M$ which satisfy the condition of Lemma 9.1 and that $Q(\Lambda) \leqq \tilde{\varepsilon}$ for $\Lambda \equiv\{(x, t) \mid t \geqq T\}$. That $Q(\Lambda)$ is small for large $T$ follows from the finiteness of $Q\left(\Lambda_{0}\right)$ [cf. (9.2)], $\Lambda_{0} \equiv\{(x, t) \mid t \geqq 0\}$. We define $\chi_{i}^{1}, \chi_{i}^{2}$ and $T^{*}$ as in Lemma 9.1. Let $u_{ \pm}^{j}(s), j=1,2$, be the one-sided limits of $u(x, t)$ along $\chi_{i}^{j}$ at time s. Suppose that $\chi_{i}^{1}$ and $\chi_{i}^{2}$ enters $\Omega_{i}$ before time $\tilde{T} \geqq T^{*}$. Because of our choice of $T$, we have $\left|u_{-}^{1}(s)-v_{i-1}\right|+\left|u_{+}^{2}(s)-v_{i}\right|$ $=O(1)(\varepsilon+\tilde{\varepsilon})$ for $s \geqq \tilde{T}$. Thus it follows from (9.7) that

$$
\begin{aligned}
\frac{d D_{i}(s)}{d s} & \leqq \theta Y_{i}^{+}(s)+O(1) \tilde{\chi}_{i}(s)+(1-\theta)\left(\lambda_{i}\left(v_{i}\right)-\lambda_{i}\left(v_{i-1}\right)+O(1)(\varepsilon+\tilde{\varepsilon})\right. \\
& =\theta Y_{i}^{+}(s)+(1-\theta)\left(\lambda_{i}\left(v_{i}\right)-\lambda_{i}\left(v_{i-1}\right)+O(1)(\varepsilon+\tilde{\varepsilon}), \quad s \geqq T\right.
\end{aligned}
$$

for some constant $0<\theta<1$. Thus from (9.5) we have the following integral inequality for $D_{i}(s)$ :

$$
\frac{d D_{i}(s)}{d s} \leqq \theta \frac{D_{i}(s)}{s-T^{*}}+(1-\theta)\left(\lambda_{i}\left(v_{i}\right)-\lambda_{i}\left(v_{i-1}\right)+O(1)(\varepsilon+\tilde{\varepsilon}), \quad s \geqq \tilde{T},\right.
$$

and so

$$
D_{i}(s) \leqq\left(\lambda_{i}\left(v_{i}\right)-\lambda_{i}\left(v_{i-1}\right)\left(s-T^{*}\right)+O(1)(\varepsilon+\tilde{\varepsilon})\left(s-T^{*}\right) .\right.
$$

Since $\lambda_{i}\left(v_{i}\right)-\lambda_{i}\left(v_{i-1}\right)<0$ and $\varepsilon, \tilde{\varepsilon}$ are arbitrary, we conclude from (19.5) that $D_{i}(s) \equiv 0$ for $s$ and $T$ sufficiently large. That is, $\chi_{s}^{1}$ and $\chi_{i}^{2}$ coalesce to form an $i$-shock wave and outside the shock wave, $u(x, t) / \Omega_{i}$ has total variation $O(1) Q(\Lambda)+\varepsilon$ $=O(1)(\varepsilon+\tilde{\varepsilon})$. In particular we show that $v_{i} \in S_{i}\left(v_{i-1}\right)$.

Similar arguments also yield that if $\lambda_{i}\left(v_{i}\right) \geqq \lambda_{i}\left(v_{i-1}\right)$, $i$-field g.nl., then $u(x, t) / \Omega_{i}$ tends to an $i$-rarefaction wave and $v_{i} \in R_{i}\left(v_{i-1}\right)$. Thus we have shown that $\left\{v_{0}\right.$, $\left.v_{1}, \ldots, v_{n}, \quad v_{ \pm}\right\}$satisfies $(8.1) \sim(8.3)$. This completes the proof of the theorem. Q.E.D.

\section{Strong Asymptotic Behavior}

In this section we assume that

$$
\begin{gathered}
u_{0}(x)=\left\{\begin{array}{lll}
u_{l} & \text { for } & x<-M, \\
u_{r} & \text { for } & x>M,
\end{array}\right. \\
g(x, u) \equiv 0 \text { for }|x|>M
\end{gathered}
$$

for some positive constant $M$ and constant states $u_{l}$ and $u_{r}$.

Theorem 11.1. Suppose that the hypothesis of Theorem 10.1 and the assumption (11.1) hold. Then the convergence of the solution $u(x, t)$ of $(0.1)$ and $(0.2)$ to simple waves as described in i) $\sim \mathrm{v}$ ) of Theorem 10.1 attains the following rates: the convergence to shock waves, steady state solution and traveling waves is of the rate $t^{-3 / 2} ;$ the convergence to rarefaction waves is of the rate $t^{-1 / 2}$, and the amount of $i$ waves in $\Omega_{j}, i \neq j$ tends to zero at the rate of $t^{-3 / 2}$. 
Proof. According to Theorem 10.1, there exists $T_{0}>0$ such that the amount of $i$ waves in $\Omega_{j}$ and the amount of wave interactions is small after time $T_{0}$ as compared to the strength of relatively strong shock waves [cf. v), Theorem 10.1] that have imerged from the solution before time $T_{0}$. Theorem 10.1 does not provide an estimate for $T_{0}$, however, the techniques used in its proof and to be used in this proof can be applied to obtain an estimate for $T_{0}$. In general, $T_{0}$ is large if the minimum strength of the shock waves in $\left(u_{i-1}, u_{i}\right)$ (cf. Lemma 9.1) is small. We will not elaborate on this point however (cf. [18]). Suppose that $\left(u_{i-1}, u_{i}\right)$ is a shock wave with strength $\alpha_{i}$ for $i \in\left\{s_{1}, s_{i}, \ldots, s_{p}\right\} \subset\{1,2, \ldots, n\}$, and $\left(u_{i-1}, u_{i}\right)$ is a rarefaction wave for $i \in\left\{r_{1}, r_{2}, \ldots, r_{q}\right\} \subset\{1,2, \ldots, n\}$, and $\left(u_{i-1}, u_{i}\right)$ is a contact discontinuity for $i \notin\left\{s_{1}, \ldots, s_{p}, r_{1}, \ldots, r_{q}\right\}$. The strengths of the relatively strong shock waves in $\Omega_{i}$, $i=s_{1}, s_{2}, \ldots, s_{q}$, are denoted by $\alpha_{i}(t)$, so that $\alpha_{i}(t)$ is close to $\alpha_{i}$ for $t$ large. We will use $(4.1)_{4}$ to define $Q_{i}^{s}$ for $i=r_{1}, r_{2}, \ldots, r_{q}$ and $(4.1)_{5}$ to define $Q_{i}^{s}$ for $i=s_{1}, s_{2}, \ldots, s_{p}$, and show by induction that

$$
\begin{aligned}
& \max \left\{\left|X_{i}^{-}(t)\right|, i=r_{1}, r_{2}, \ldots, r_{q}\right\}=O(1) t^{-1 / 2}, \\
& Q(t)=O(1) t^{-3 / 2} \text { as } t \rightarrow+\infty
\end{aligned}
$$

For a given $t \geqq M$, assumption (11.1) implies that there exist $x_{l}(t), x_{r}(t), \mid x_{l}(t)$ $-x_{r}(t) \mid=O(1) t$, such that $g \equiv 0$ for $x>x_{r}(t)$ or $x<x_{l}(t)$, and $u(x, t)$ equals $u_{l}$ for $x<x_{l}(t)$ and equals $u_{r}$ for $x>x_{r}(t)$. Through $\left(x_{l}(t), t\right)$ and $\left(x_{r}(t), t\right), t \geqq M$, respectively, we draw $i$-characteristics $\chi_{i}^{1}(t)$ and $\chi_{i}^{2}(t)$. Because $\lambda_{i}(u(x, t)), i=1,2, \ldots, n$, are nonzero and distinct, there exists $t^{*}>t$, such that $\chi_{j}^{1}(t), \chi_{j}^{2}(t)$ do not intersect $\chi_{k}^{1}(t), \chi_{k}^{2}(t), k \neq j$, and these characteristics leave the region $|x|<M$ after time $t^{*}$. Moreover, for some $C>1$ independent of $t$,

$$
t^{*} \leqq C t
$$

Note that in the present situation we may take $\varepsilon$ in Lemmas 9.1 and 9.2 to be zero. We set $\theta=T V+G_{1}, T V$ total variation in $x$ of $u(x, 0)$ and $G_{1}$ the $L_{1}(x)$ norm of $\max _{x, u}\left\{\mathrm{~g}, \frac{\partial g}{\partial u}\right\}$. We have assumed $\theta$ to be small.

We shall prove by induction that for

$$
T_{0}=\theta^{-11 / 5}\left(x_{r}(T)-X_{l}(T)+1\right),
$$

there exist sequences $\left\{K_{m}\right\},\left\{L_{m}\right\}$, and $\left\{\gamma_{m}\right\}, m=0,1,2, \ldots$, such that

$$
\begin{aligned}
& \max \left\{\left|X_{i}^{-}(t)\right|, i=r_{1}, r_{2}, \ldots, r_{q}\right\} \leqq K_{m} t^{-1 / 2}, \quad \text { for } t \leqq C^{m} T_{0} \\
& Q(t) \leqq L_{m} t^{-3 / 2} \quad \text { for } t \leqq C^{m} T_{0}, \quad m=0,1,2, \ldots \\
& K_{m}=K_{m-1}(2 C)^{O(1) \gamma_{m-1}}\left[1+O(1) \gamma_{m-1}+O(1) 2^{(-(m-1) / 10)} \theta^{1 / 5}\right] \quad m=1,2, \ldots \\
& \gamma_{m} \leqq 2^{-1 / 10} \gamma_{m-1}<1, \quad m=1,2, \ldots \\
& L_{m}=O(1)\left(K_{m}\right)^{3}, \quad m=0,1,2, \ldots
\end{aligned}
$$


These estimates imply that $K=\varlimsup_{m \rightarrow+\infty} K_{m}$ and $L=\varlimsup_{m \rightarrow+\infty} L_{m}$ exist and are finite and thus yield (11.2) and (11.3). To start the induction process we set

$$
K_{0}=\theta T_{0}^{1 / 2}, \quad L_{0}=K_{0}^{3},
$$

so that (11.4) (11.6) hold for $m=0$ if $T_{0}$ is large (Theorem 10.1). Suppose that (11.4) (11.8) hold for $m=p \geqq 1$. We set

$$
T_{*}=\left(K_{p} C^{p} T_{0}\right), \quad \gamma_{p}=K_{p} \tilde{T}^{-1 / 2}
$$

so the induction hypothesis implies that

$$
\begin{aligned}
& \max \left\{\left|X_{i}^{-}(t)\right|, j=r_{1}, r_{2}, \ldots, r_{q}\right\} \leqq \gamma_{p}, \\
& Q(t) \leqq L_{p} T_{*}^{-3 / 2}=O(1) \gamma_{p}^{3}, \quad 0 \leqq t \leqq T_{*} .
\end{aligned}
$$

Let $x_{i}(t)$ be any point between $\chi_{i}^{2}\left(T_{*}\right)$ and $\chi_{i-1}^{1}\left(T_{*}\right)$. Because of the presence of a strong $i$-shock wave, $i=s_{1}, s_{2}, \ldots, s_{q}, \chi_{i}^{1}\left(C^{p} T_{0}\right)$ and $\chi_{i}^{2}\left(C^{p} T_{0}\right)$ meet before a finite time $\tilde{t}$. We have from (11.10) and (11.11) (cf. proof of Theorem 10.1)

$$
\sum_{i=1}^{p}\left|X_{s_{i}}(t)-\right| \alpha_{s_{i}}(t)||+\sum_{i=1}^{n} \tilde{Y}(t)=O(1) \gamma_{p}^{3}, \quad t \geqq \tilde{t},
$$

where $\tilde{Y}(t)$ is the total amount of $j$-waves, $j=1,2, \ldots, n$, not between $\chi_{i}^{1}\left(T^{*}\right)$ and $\chi_{i}^{2}\left(T^{*}\right)$. This estimate and (11.10), (11.11) imply that

$$
\left|u\left(x_{i}, t\right)-u_{i}\right|=O(1) \gamma_{p}^{3} \quad \text { for } t \geqq C T_{*} .
$$

For $i=r_{1}, r_{2}, \ldots, r_{q}$, it follows from (11.11) and (11.13) that

$$
\begin{aligned}
& Y_{i}^{+}(t)+Y_{i}^{-}(t)+\operatorname{Str} \cdot \chi_{i}^{1}\left(T^{*}, t\right)+\operatorname{Str} \cdot \chi_{i}^{2}\left(T^{*}, t\right) \\
& \quad=\left(u_{l}, u_{r}\right)_{i}+O(1) \gamma_{p}^{3},
\end{aligned}
$$

where $Y_{i}^{+}(t)$ and $Y_{i}^{-}(t)$ are the amount of $i$-waves between $\chi_{i}^{1}\left(T_{*}\right)$ and $\chi_{i}^{2}\left(T_{*}\right)$ at time t. It follows from (1.1) and (9.6) that, for $C T_{*} \leqq T \leqq C^{p} T_{0}$,

$$
\begin{aligned}
\frac{d D_{i}(t)}{d t}= & \lambda_{-}^{2}(t)-\lambda_{+}^{1}(t)+\frac{1}{2}\left(\operatorname{Str} \cdot \chi_{i}^{1}\left(T^{*} ; t\right)+\operatorname{Str} \cdot \chi_{i}^{2}\left(T^{*} ; t\right)\right) \\
& +O(1)\left[\operatorname{Str} \cdot \chi_{i}^{1}\left(T^{*} ; t\right)+\operatorname{Str} \cdot \chi_{i}^{2}\left(T^{*} ; t\right)\right]^{2},
\end{aligned}
$$

whence we have from (11.10). (11.11), and (11.14) that

$$
\frac{d D_{i}(t)}{d s} \leqq K_{p} t^{1 / 2}\left(\frac{1}{2}+O(1) \gamma_{p}\right)+O(1) \gamma_{p}^{3}+\left(u_{l}, u_{r}\right)_{i}, \quad C T_{*} \leqq t \leqq C^{p} T_{0} .
$$

Integrating this inequality from $C T_{*}$ to $C^{p} T_{0}$, we obtain

$$
\begin{aligned}
D_{i}\left(C^{p} T_{0}\right) \leqq & \frac{1}{2}\left(1+O(1) \gamma_{p}\right) K_{p}\left[\left(C^{p} T_{0}\right)^{1 / 2}-\left(C T_{*}\right)^{1 / 2}\right] \\
& +O(1) \gamma_{p}^{3}\left(C^{p} T_{0}+D_{i}\left(C T_{*}\right)+\left(u_{l}, u_{r}\right)_{i}\left(C^{p} T_{0}-C T_{*}\right) .\right.
\end{aligned}
$$


For $C^{p} T_{0} \leqq t \leqq C^{p+1} T_{0}$, we use (11.10), (11.11), (11.14), and (9.5) to conclude:

$$
\frac{d D_{i}(t)}{d t} \leqq\left(\frac{1}{2}+O(1) \gamma_{p}\right) \frac{D_{i}(t)}{t-C T_{*}}+O(1) \gamma_{p}^{3}+\left(u_{l}, u_{r}\right)_{i} \text {. }
$$

By solving this differential inequality, we have from (11.16) that

$$
\begin{gathered}
D_{i}(t) \leqq \frac{1}{2}\left[\left(1+O(1) \gamma_{p}\right) K_{p}\left(\left(C^{p} T_{0}\right)^{1 / 2}-\left(C T_{*}\right)^{1 / 2}\right)+O(1) \gamma_{p}^{3} C^{p} T_{0}+O(1) T_{*}\right], \\
{\left[\frac{t-C T_{*}}{C^{p} T_{0}-C T_{*}}\right]^{1 / 2+O(1) \gamma_{p}}+\left[O(1) \gamma_{p}^{3}+\left(u_{l}, u_{r}\right)_{i}\right]\left(t-C T_{*}\right) .}
\end{gathered}
$$

This estimate along with (9.5), (11.10), and (11.13) yields estimates for $Y_{i}^{ \pm}(t)$. The desired estimate for $X_{i}^{-}(t)$ in (11.4), $K_{p+1}$ in (11.6), and $\gamma_{p+1}$ in (11.7) then follows from (11.12) and (11.9). Details are omitted.

We now prove (11.5) and (11.8) for $m=p+1$. Using (9.4) repeatedly we have from (11.5) for $m \leqq p$ that

$$
\begin{aligned}
Q(t) & =Q_{0}^{s}(t)+\sum_{k=1}^{p}(O(1) \theta)^{k} Q_{0}^{s}\left(C^{-k} t\right)+(O(1) \theta)^{p} Q\left(C^{-p} t\right) \\
& =Q_{0}^{s}(t)+O(1) \sum_{k=1}^{p}(O(1) \theta)^{k} K_{p}^{3}\left(C^{-k} t\right)^{-3 / 2}, C^{p} T_{0} \leqq t \leqq C^{p+1} T_{0} .
\end{aligned}
$$

For $i=r_{1}, r_{2}, \ldots, r_{q}$, we have from (11.4) for $m \leqq p+1$, which has just been proved, that

$$
Q_{s}^{i}(t)=O(1)\left|X_{i}^{-}(t)\right|^{3} \leqq O(1) K_{p+1}^{3} t^{-3 / 2}, \quad C^{p} T_{0} \leqq t \leqq C^{p+1} T_{0} .
$$

For $i=s_{1}, s_{2}, \ldots, s_{p}$, and $C^{p} T_{0} \leqq t \leqq C^{p+1} T_{0}$, there exists $\tilde{t}<t$ such that $\chi_{i}^{1}(\tilde{t})$ and $\chi_{i}^{2}(\tilde{t})$ meet before time $t$ and $t \leqq O(1) \tilde{t} / \alpha_{s_{i}}$. This is so because of the presence of the relatively strong shock wave. Thus it follows from the induction hypothesis (11.12) and (4.1) that

$$
\begin{aligned}
Q_{s}^{i}(t) & =O(1)\left[\left|X_{i}(t)-\alpha_{s_{i}}(t)\right|\left(\alpha_{s_{i}(t)} 2_{+}\left|X_{i}(t)-\alpha_{s_{i}}(t)\right|^{3}\right]\right. \\
& =O(1)\left[Q(\tilde{t})\left(\alpha_{s_{i}}(t)\right)^{2}+(Q(\tilde{t}))^{3}\right] \\
& =O(1)\left(K_{p+1}\right)^{3}\left[\left(\alpha_{s_{i}} t\right)^{-3 / 2}\left(\alpha_{s_{i}}(t)\right)^{2}+\left(\alpha_{s_{i}} t\right)^{-3 / 2} Q(\tilde{t})^{2}\right] \\
& =O(1)\left(K_{p+1}\right)^{3} t^{-3 / 2}\left[\left(\alpha_{s_{t}}\right)^{1 / 2}+\left(\alpha_{s_{i}}\right)^{-3 / 2} Q(\tilde{t})^{2}\right]
\end{aligned}
$$

which is $O(1)\left(K_{p+1}\right)^{3} t^{-3 / 2}$ because $Q(\tilde{t})$ is small. Finally (11.5) and (11.8) for $m=p+1$ follow from (11.17) (11.19). This completes the proof of (11.2) and (11.3). The theorem now follows from (11.2) and (11.3) using similar techniques used in the proof of Theorem 10.1. Details are omitted. Q.E.D.

Suppose that $u_{l}=u_{0}(-\infty)$ and $u_{r}=u_{0}(+\infty)$ are related by a steady state solution $u(x)$ of $(0.6)$, i.e., $u=u(-\infty)$ and $u_{r}=u(+\infty)$. Then in Theorem $8.1, u_{i}=u_{l}$ for $i=1,2, \ldots, p$ and $u_{i}=u_{r}$ for $i=p+1, \ldots, n$, and Theorem 11.1 implies that the 
solution $u(x, t)$ of $(0.1)$ and $(0.2)$ approaches the steady state solution $u(x)$ of $(0.6)$ and traveling waves uniformly and in total variation norm at the rate $t^{-1 / 2}$ as $t$ tends to infinity. In fact, more is true: $u(x, t)$ tends to a linear superposition of $N$ waves, traveling waves and steady state solution in $L_{1}(x)$ norm and the convergence is also at algebraic rates $[17,18]$.

Acknowledgement. I want to thank Professor James Glimm whose interest in the problem has been a great encouragement.

\section{References}

1. Antman, S., Liu, T.-P.: Traveling waves in hyperelastic rods. Quart. Appl. Math. (to appear)

2. Chester, W.: The quasi-cylindrical shock tube. Philos. Mag. (7) 45, 1293-1304 (1954)

3. Chisnell, P.: The normal motion of a shock wave through a nonuniform one-dimensional medium. Proc. R. Soc. Edinburgh Sect. A 323, 350-370 (1955)

4. Conley, C.C., Smoller, J.A.: Shock waves as limits of progressive wave solutions of higher order equation. Comm. Pure Appl. Math. 24, 459-472 (1971)

5. Courant, R., Friedrichs. K.O.: Supersonic flow and shock waves. New York: Interscience (1948)

6. Courant, R., Hilbert, D.: Methods of mathematical physics. Vol. II, Chapt. V. 6, pp. 464-471. Interscience Publishers (1962)

7. Dafermos, C.M. : The entropy rate admissibility criterion for solutions of hyperbolic conservation laws. J. Diff. Eq. 14, 202-212 (1973)

8. DiPerna, R.: Decay and asymptotic behavior of solutions to nonlinear hyperbolic systems of conservation laws. Indiana Univ. Math. J. 24, 1047-1071 (1975)

9. Glimm. J.: Solutions in the large for nonlinear hyperbolic systems of equations. Comm. Pure Appl. Math. 18, 697-715 (1965)

10. Glimm, J., Lax, P.D.: Decay of solutions of nonlinear hyperbolic conservation laws. Am. Math. Soc. 101, (1970)

11. John, F.: Formation of singularities in one-dimensional nonlinear wave propagation. Comm. Pure Appl. Math. 27, 377-405 (1974)

12. Lax, P.D.: Hyperbolic systems of conservation laws. II. Comm. Pure Appl. Math. 10, 537-566 (1957)

13. Lax, P.D.: Shock waves and entropy. In: Contributions to nonlinear functional analysis. Zarantonello, E.H. (ed.), pp. 603-634. New York: Academic Press 1971

14. Lax, P.D.: Development of singularities of solutions of nonlinear hyperbolic partial differential equations. J. Math. Phys. 5, 611-613 (1964)

15. Liu, T.-P.: The Riemann problem for general systems of conservation laws. J. Diff. Eq. 18, 218-234 (1975)

16. Liu, T.-P.: Solutions in the large for equations of non-isotropic gas dynamics. Indiana Univ. J. 26, 147177 (1977)

17. Liu, T.P.: Decay to $N$-waves of solutions of general systems of nonlinear hyperbolic conservation laws. Comm. Pure Appl. Math. 30, 585-610 (1977)

18. Liu, T.-P.: Linear and nonlinear large-time behavior of solutions of general systems of hyperbolic conservation laws. Comm. Pure Appl. Math. 30, 767-796 (1977)

19. Liu, T.-P.: The deterministic version of the Glimm scheme. Comm. Math. Phys. 57, 135-148 (1977)

20. Liu, T.-P.: The development of singularities in the nonlinear waves for quasi-linear hyperbolic partial differential equations. J. Diff. Eq. (to appear)

21. Nishida, T.: Global solutions for an initial boundary value problem of a quasilinear hyperbolic system. Proc. Jpn. Acad. 44, 642-646 (1968)

22. Nishida, T., Smoller, J.A. : Solutions in the large for some nonlinear hyperbolic conservation laws. Comm. Pure Appl. Math. 26, 183-200 (1973)

23. Riemann, B.: Über die Fortpflanzung ebener Luftwellen von endlicher Schwingungsweite. Gottinger Abhandlungen, Vol. 8, p.43. Werke, Zte Aufl. Leipzig 157 (1892) 
24. Wendroff, B.: Shock propagation in variable area ducts with phase changes: an extention of Chisnell's method. J. Eng. Math. 11, 273-286 (1977)

25. Whitham, B. : Linear and nonlinear waves. New York: John Wiley 1974

26. Concus, P., Proskurowski, W.: Numerical solution of a nonlinear hyperbolic equation by the random choice method (to appear in J. Comp. Phys.)

27. Hoffman, A.L.: A single fluid model for shock formation in MHD shock tubes. J. Plasma Phys. 1, 192-207 (1967)

Communicated by J. Glimm

Received November 11, 1978 